\title{
Molecular characterization of selectively vulnerable neurons in Alzheimer's Disease
}

Kun Leng ${ }^{1,2,3,4,13}$, Emmy Li ${ }^{1,2,3,13}$, Rana Eser ${ }^{5}$, Antonia Piergies ${ }^{5}$, Rene Sit $^{2}$, Michelle Tan ${ }^{2}$, Norma Neff ${ }^{2}$, Song Hua Li ${ }^{5}$, Roberta Diehl Rodriguez ${ }^{6}$, Claudia Kimie Suemoto ${ }^{7,8}$, Renata Elaine Paraizo Leite ${ }^{7}$, Carlos A. Pasqualucci ${ }^{7}$, William W. Seeley ${ }^{5,11}$, Salvatore Spina ${ }^{5}$, Helmut Heinsen $^{7,9}$, Lea T. Grinberg ${ }^{5,7,10,11} *$, Martin Kampmann ${ }^{1,2,12} *$

${ }^{1}$ Institute for Neurodegenerative Disease, University of California, San Francisco, San Francisco, CA, USA.

${ }^{2}$ Chan Zuckerberg Biohub, San Francisco, CA, USA.

${ }^{3}$ Biomedical Sciences Graduate Program, University of California, San Francisco, San Francisco, CA, USA.

${ }^{4}$ Medical Scientist Training Program, University of California, San Francisco, San Francisco, CA, USA.

${ }^{5}$ Memory and Aging Center, Weill Institute for Neurosciences, Department of Neurology, University of California, San Francisco, San Francisco, CA, USA.

${ }^{6}$ Department of Neurology, Universidade de São Paulo, Faculdade de Medicina, São Paulo, Brazil.

${ }^{7}$ Department of Pathology, Universidade de São Paulo, Faculdade de Medicina, São Paulo, Brazil.

${ }^{8}$ Division of Geriatrics, Department of Clinical Medicine, Universidade de São Paulo, Faculdade de Medicina, São Paulo, Brazil.

${ }^{9}$ Department of Psychiatry, University of Würzburg, Würzburg, Germany.

${ }^{10}$ Global Brain Health Institute, University of California, San Francisco, San Francisco, CA, USA.

${ }^{11}$ Department of Pathology, University of California, San Francisco, San Francisco, CA, USA.

${ }^{12}$ Department of Biochemistry and Biophysics, University of California, San Francisco, San Francisco, CA, USA.

${ }^{13}$ These authors contributed equally: Kun Leng, Emmy Li.

*e-mail: lea.grinberg@ucsf.edu; martin.kampmann@ucsf.edu

\section{ABSTRACT}

Alzheimer's disease (AD) is characterized by the selective vulnerability of specific neuronal populations, the molecular signatures of which are largely unknown. To identify and characterize selectively vulnerable neuronal populations, we used single-nucleus RNA sequencing to profile the caudal entorhinal cortex and the superior frontal gyrus - brain regions where neurofibrillary inclusions and neuronal loss occur early and late in AD, respectively - from postmortem brains spanning the progression of AD-type tau neurofibrillary pathology. We identified RORB as a marker of selectively vulnerable excitatory neurons in the entorhinal cortex, and subsequently validated their depletion and selective susceptibility to neurofibrillary inclusions during disease progression using quantitative neuropathological methods. We also discovered an astrocyte subpopulation, likely representing reactive astrocytes, characterized by decreased expression of genes involved in homeostatic functions. Our characterization of selectively vulnerable neurons in AD paves the way for future mechanistic studies of selective vulnerability and potential therapeutic strategies for enhancing neuronal resilience. 
Selective vulnerability is a fundamental feature of neurodegenerative diseases, in which different neuronal populations show a gradient of susceptibility to degeneration ${ }^{1,2}$. Selective vulnerability at the network level has been extensively explored in Alzheimer's disease (AD) ${ }^{3-5}$, currently the leading cause of dementia and lacking in effective therapies. However, little is known about the mechanisms underlying selective vulnerability at the cellular level in AD, which could provide insight into disease mechanisms and lead to therapeutic strategies.

The entorhinal cortex (EC), an allocortex, is one of the first cortical brain regions to exhibit neuronal loss in $\mathrm{AD}^{6}$. Neurons in the external EC layers, especially in layer II, accumulate taupositive neurofibrillary inclusions and die early in the course of $\mathrm{AD}^{7-12}$. However, these selectively vulnerable neurons have yet to be characterized extensively at the molecular level. Furthermore, it is unknown whether there are differences in vulnerability among subpopulations of these neurons. Although rodent models of AD have offered important insights ${ }^{13-15}$, the available models fail to capture some critical disease processes simultaneously, such as the accumulation of neurofibrillary inclusions and neuronal loss ${ }^{16}$, limiting the extrapolation of findings from rodent models to address selective vulnerability.

Previous studies have combined laser capture microdissection with microarray analysis of gene expression $^{17,18}$ to characterize EC neurons in AD, but focused on disease-related changes in gene expression, rather than selective vulnerability. More recently, single-nucleus RNA-sequencing (snRNA-seq) has enabled large-scale characterization of transcriptomic profiles of individual cells from post-mortem human brain tissue ${ }^{19,20}$. However, snRNA-seq studies of AD published to date have focused on cell-type specific differential gene expression between AD cases and healthy controls ${ }^{21,22}$, without explicitly addressing selective vulnerability.

Here, we performed snRNA-seq on post-mortem brain tissue from a cohort of cases spanning the progression of AD-type tau neurofibrillary pathology to characterize changes in the relative abundance of cell types and cell type subpopulations. Importantly, we discovered a selectively vulnerable subpopulation of excitatory neurons in the entorhinal cortex and validated the selective depletion of this subpopulation during $\mathrm{AD}$ progression with quantitative histopathology, using multiplex immunofluorescence in EC regions delineated by rigorous cytoarchitectonic criteria. In addition, we examined subpopulations of inhibitory neurons, which did not show differences in vulnerability, and also subpopulations of microglia, oligodendrocytes, and astrocytes. We uncovered an astrocyte subpopulation likely corresponding to reactive astrocytes that showed downregulation of genes involved in homeostatic function.

\section{RESULTS}

\section{Cohort selection and cross-sample alignment}

We performed snRNA-seq on cell nuclei extracted from postmortem brain tissue (see Methods) from the entorhinal cortex (EC) at the level of the mid-uncus and from the superior frontal gyrus (SFG) at the level of the anterior commissure (Brodmann area 8), from 10 male APOE $\varepsilon 3 / \varepsilon 3$ 
individuals representing the cortical-free, early and late stages of AD-type tau neurofibrillary pathology (Braak stages ${ }^{3}$ 0, 2 and 6; Fig. 1a, Table 1).

The neuropathological hallmarks of AD are amyloid plaques, which are measured by the CERAD scores ${ }^{23}$ and Thal phases ${ }^{24}$, and neurofibrillary inclusions consisting of hyperphosphorylated tau protein (phospho-tau) aggregates, which are measured by the Braak staging system $^{3}$. The Braak staging system is based on the stereotypical topographical progression of neurofibrillary inclusions to different brain regions. Neurofibrillary inclusions are first found in specific subcortical structures in the brainstem (Braak stages a-c, also hereon referred to collectively as Braak stage 0). Subsequently, the transentorhinal and entorhinal cortices, followed by the hippocampal formation, are the first areas of the cerebral cortex to accumulate tau pathology (Braak stages 1-2). The limbic areas and temporal neocortex then follow (Braak stages 3-4), and finally, other neocortical association areas (such as the SFG) and primary neocortical areas are involved (Braak stages 5-6) ${ }^{3,25}$. Since the accumulation of neurofibrillary inclusions is the best correlate of clinical cognitive decline, after neuronal loss ${ }^{26}$, we reasoned that profiling matched EC and SFG samples across different Braak stages would allow us to isolate the effect of disease progression on cell types and cell type subpopulations.

A challenge in characterizing the impact of disease progression on different cell type subpopulations is that these subpopulations need to be defined in a way that is independent from the effect of disease progression. Typically, cell type subpopulations are defined by sub-grouping cells of the same cell type through cluster analysis (i.e. clustering), followed by examination of marker gene expression in the resulting clusters. To remove the effect of disease progression on clustering, we performed, prior to clustering, cross-sample alignment ${ }^{27-29}$ of the data from each brain region using scAlign (see Methods), which learns a low-dimensional manifold (i.e. the alignment space) in which cells tend to cluster in a manner consistent with their biological function independent of technical and experimental factors ${ }^{29}$. Importantly, after identifying clusters in the alignment space, we used the original data for subsequent analyses involving examination of gene expression, such as identifying differentially expressed genes between clusters.

\section{Changes in broad cell type composition with neuropathological AD progression}

After quality control (see Methods), we recovered 42,528 cells from the EC and 63,608 cells from the SFG. Examination of the average number of genes and unique molecular identifiers (UMIs) detected per cell showed similar or superior transcript coverage compared to previously published AD snRNA-seq datasets ${ }^{21,22}$ (Extended Data Fig. 1a,b).

After cross-sample alignment, we performed clustering and recovered 13 clusters in the EC and 18 clusters in the SFG. In both brain regions, clusters demonstrated spatial grouping in tstochastic neighborhood embedding (tSNE) that was largely uncorrelated with the individual of origin (Fig. 1b,c). Furthermore, clusters showed specific expression of cell type markers and grouped in a manner consistent with their expression of cell type markers in hierarchical clustering (Fig. 1d,e, see Methods). For comparison, we also performed clustering without crosssample alignment, which resulted in many clusters that were defined by individual of origin in 
addition to cell type (Extended Data Fig. 1c-f). Having confirmed the effectiveness of crosssample alignment in removing the effect of technical and experimental factors on clustering, we then assigned clusters to broad cell types (i.e. excitatory neurons, inhibitory neurons, astrocytes, oligodendrocytes, oligodendrocyte precursor cells, microglia, and endothelial cells) based on their expression of cell type markers (Fig. 1d,e, see Methods).

Next, to assess whether the proportions of broad cell types in the EC and SFG change with disease progression, we aggregated clusters assigned to the same cell type for each individual and then computed the relative abundance of each cell type in each individual. We tested the statistical significance of changes in relative abundance using beta regression ${ }^{30}$ (see Methods), which is suitable for variables ranged from 0 to 1 . After correcting for multiple testing (Holm's method, threshold for significant adjusted $P$-values set at 0.05; see Methods), we found statistically significant increases in the relative abundance of endothelial cells in the EC (Fig. 1f) in Braak stages 2 and 6 compared to Braak stage 0, although the magnitude of the changes were small. We also found a trend towards increased relative abundance of microglia in the EC in Braak stage $6\left(P_{\text {adjusted }}=0.08\right)$, suggestive of microgliosis. In the SFG, however, we did not observe an upward trend in the relative abundance of microglia with disease progression (Fig. $1 \mathrm{~g})$. As for other broad cell types, we did not detect changes in relative abundance that were statistically significant after correction for multiple hypothesis testing. However, we observed a downward trend in the relative abundance of EC excitatory neurons in Braak stages 2 ( $P_{\text {unadjusted }}$ $=0.18)$ and $6\left(P_{\text {unadjusted }}=0.02\right)$, and of SFG excitatory neurons only in Braak stage $6\left(P_{\text {unadjusted }}=\right.$ 0.05), consistent with early involvement of the EC and sparing of the SFG until late Braak stages, and the previously described greater vulnerability of excitatory neurons relative to inhibitory neurons in $\mathrm{AD}^{15,31}$.

\section{Selective vulnerability of excitatory neuron subpopulations}

Previous single-cell transcriptomic studies of human and mouse cortex have shown that unbiased clustering of excitatory neurons largely recapitulates the laminar organization of the cortex ${ }^{19,20}$. In the context of $\mathrm{AD}$, tau neurofibrillary inclusions are known to preferentially accumulate in neocortical layers III and $\mathrm{V}^{3,32,33}$, most likely reflecting the selective vulnerability of specific neuronal subpopulations. Therefore, we asked whether specific excitatory neuron subpopulations show a decline in their relative abundance with disease progression, by performing subclustering of excitatory neurons in the EC and SFG after cross-sample alignment (see Methods).

In the EC, we discerned nine excitatory neuron subpopulations (Fig. 2a-d). These subpopulations exhibited distinct expression of EC layer-specific genes identified in the mouse medial EC ${ }^{34}$, which phylogenetically resembles the human caudal $\mathrm{EC}^{35,36}$. Notably, subpopulation EC:Exc.s2 showed a striking $~ 50 \%$ decrease in its relative abundance in Braak stage 2 compared to Braak stage 0, with no further decrease in Braak stage 6 (Fig. 2c), suggesting depletion early in disease. EC:Exc.s1 and EC:Exc.s4 similarly exhibited a $\sim 50-60 \%$ reduction in their relative abundance in Braak stage 2. EC:Exc.s1, EC:Exc.s2, and EC:Exc.s4 expressed genes associated with mouse EC layer II (Fig. 2c), consistent with the fact that tau neurofibrillary inclusions are known to accumulate preferentially in human EC layer II early in $\mathrm{AD}^{7-10}$. However, not all subpopulations expressing genes associated with mouse EC layer II showed similar levels of early vulnerability. For example, EC:Exc.s6 and EC:Exc.s8 did not demonstrate statistically significant changes in 
their relative abundance across disease progression. Outside of EC layer II, we failed to find evidence of selective vulnerability in neuronal subpopulations expressing genes associated with mouse EC layer III (EC:Exc.s0) or V/VI (EC:Exc.7, EC:Exc.s5, EC:Exc.s3). In fact, EC:Exc.s5 exhibited a statistically significant increase in its relative abundance in Braak stage 2. Since neurons are post-mitotic, this increase is likely due to the selective earlier depletion of more vulnerable excitatory neuron subpopulations, followed by later depletion of EC:Exc.s5.

To identify molecular markers of selectively vulnerable excitatory neuron subpopulations in the EC (EC:Exc.s2, EC:Exc.s4, EC:Exc.s1), we inspected transcript levels of genes differentially expressed between pairs of subpopulations and curated a set of genes which were specifically expressed by no more than four subpopulations (Extended Data Fig. 2a), which we decided was a reasonable threshold for a positive marker to be useful. We found that EC:Exc.s2 and EC:Exc.s4 specifically expressed RORB, CTC-340A15.2 and CTC-535M15.2 (Fig. 2c). RORB (RAR-related Orphan Receptor B) encodes a transcription factor known as a marker and developmental driver of layer IV neurons in the neocortex ${ }^{37-39}$, but is also expressed by neurons in other layers ${ }^{20}$. Little is known about the non-coding transcripts CTC-340A15.2 and CTC$535 M 15.2$ in the context of neuronal identity and function. We also found that EC:Exc.s1 was marked by high expression of $C D H 9$ (Fig. 2c), a cadherin with neuron-specific expression. However, $\mathrm{CDH} 9$ was also expressed by other excitatory neuron subpopulations in the EC, and we could not find markers that were specifically expressed only in EC:Exc.s1. Therefore, we chose to focus our analysis on EC:Exc.s2 and EC:Exc.s4.

In addition to identifying molecular markers of the selectively vulnerable EC:Exc.s2 and EC:Exc.s4 neurons, we also enumerated genes that were differentially expressed in EC:Exc.s2 and EC:Exc.s4 compared to all other excitatory neurons in the EC, controlling for differences across individuals (see Methods). We found that genes with higher expression in EC:Exc.s2 and EC:Exc.s4 were enriched for axon-localized proteins and voltage-gated potassium channels, whereas genes with lower expression in EC:Exc.s2 and EC:Exc.s4 were enriched for synapseand dendrite-localized proteins and pathways involving G-protein mediated signaling, ion transport, and neurotransmitter receptor signaling (Extended Data Fig. 2b-e, Supplementary Table 1).

We also performed differential gene expression analysis across Braak stages for EC excitatory neuron subpopulations (see Methods), choosing to focus on comparing Braak stage 6 vs. 0, which yielded the largest number of differentially expressed genes. We found a broad decrease in expression of genes encoding pre- and post-synaptic proteins in Braak stage 6 vs. 0 for many EC excitatory neuron subpopulations (Extended Data Fig. 3b,d,f). Furthermore, we observed that EC:Exc.s2, which demonstrated a statistically significant decline in relative abundance in Braak stage 6 vs. 0, also had the largest number of downwardly differentially expressed genes and the strongest enrichments for pre- and post-synaptic proteins in these genes (Extended Data Fig. $3 b, d)$. Overall, the downregulation of synapse-related genes we have observed mirrors the findings from a recent preprint by Marinaro et al. ${ }^{40}$, which examined the frontal cortex in familial monogenic AD using snRNA-seq, and is consistent with a previous study of gene expression changes in $\mathrm{AD}$ in the entorhinal cortex and other brain regions employing laser capture microdissection of neurons followed by DNA microarray analysis ${ }^{17}$. 
Having identified and characterized selectively vulnerable excitatory neuron subpopulations in the EC, we next examined excitatory neuron subpopulations in the SFG. Similar to previous studies $^{19,20}$, we found that excitatory neuron subpopulations in the SFG (11 in total) expressed distinct sets of neocortical layer-specific genes (Fig. 2b,d), recapitulating the laminar organization of the neocortex. Interestingly, SFG:Exc.s4 and SFG:Exc.s2, which were marked by $R O R B, C T C-340 A 15.2$ and $C T C-535 M 15.2$, trended towards decreased relative abundance only in Braak stage 6 (Fig. 2d; SFG:Exc.s4 $P_{\text {unadjusted }}=0.06$, SFG:Exc.s2 $P_{\text {unadjusted }}=0.36$ ), consistent with the late appearance of neurofibrillary inclusions in the SFG starting at Braak stage 5. On the other hand, SFG:Exc.s7, which was also marked by RORB, CTC-340A15.2 and CTC-535M15.2, exhibited a statistically significant but small decrease in relative abundance in Braak stage 2; however, we did not interpret this as a sign of tau pathology-associated selective vulnerability given that neurofibrillary inclusions should not be present in the SFG in Braak stage 2.

Given that SFG:Exc.s4 and SFG:Exc.s2 expressed similar markers as EC:Exc.s4 and EC:Exc.s2, we wondered if SFG:Exc.s4 and SFG:Exc.s2 may resemble EC:Exc.s4 and EC:Exc.s2 more broadly at the transcriptome level. To test this, we calculated the Pearson correlation coefficient between the expression profiles of SFG and EC subpopulations and found that SFG:Exc.s4 and SFG:Exc.s2 were indeed most similar to EC:Exc.s4 and EC:Exc.s2 (Fig. 2e). This finding is consistent with the reported similarity between deep layer neocortical excitatory neurons and EC excitatory neurons in general ${ }^{41}$. Furthermore, this correspondence was preserved when we mapped subpopulations in the EC to those in the SFG by performing cross-sample alignment for both brain regions jointly (Extended Data Fig. 4). The similarity in transcriptomes of vulnerable excitatory neurons in different brain regions is intriguing and suggests similar mechanisms of selective vulnerability in different brain regions.

Although the decrease in the relative abundance of SFG:Exc.s2 and SFG:Exc.s4 in Braak stage 6 was not statistically significant after correction for multiple testing, we asked if we could detect signs of selective vulnerability in neocortical $R O R B$-expressing excitatory neurons in an independent dataset with a larger sample size. To this end, we reanalyzed data from Mathys $e t$ $a l{ }^{21}$, which profiled the prefrontal cortex from $24 \mathrm{AD}$ cases and 24 healthy controls, with our cross-sample alignment pipeline and performed subclustering of excitatory neurons. In the Mathys et al. dataset ${ }^{21}$, we discerned 10 excitatory neuron subpopulations, each of which expressed distinct sets of neocortical layer-specific genes (Extended Data Fig. 5a,b) similar to Lake et al. ${ }^{19}$ and our dataset. Of these 10 subpopulations, Mathys:Exc.s4, Mathys:Exc.s5, and Mathys:Exc.s1 expressed RORB at high levels (CTC-340A15.2 and CTC-535M15.2 were not available in the pre-processed Mathys et al. ${ }^{21}$ data). Importantly, we observed a statistically significant decrease in the relative abundance of Mathys:Exc.s4 in male AD cases vs. controls (Extended Data Fig. 5b), recapitulating the selective vulnerability observed in our dataset, which consists only of male individuals. Furthermore, gene expression correlation analysis showed that Mathys:Exc.s4 was the most similar to EC:Exc.s2 and EC:Exc.s4 (Extended Data Fig. 5c), again demonstrating similarity between selectively vulnerable excitatory neurons in the neocortex and those in the EC.

Although we did not detect any statistically significant changes in the relative abundance of $R O R B$-expressing subpopulations in female individuals in Mathys et $a l^{21}$, Mathys.Exc.s1 trended towards decreased relative abundance in female AD cases $\left(P_{\text {unadjusted }}=0.17\right)$ and mapped 
to EC:Exc.s2 by gene expression correlation (Extended Data Fig. 5b,c). Furthermore, Marinaro et $a l .{ }^{40}$ included both male and female cases of monogenic $\mathrm{AD}$ and also reported the selective vulnerability of two out of four $R O R B$-expressing excitatory neuron subpopulations in the prefrontal cortex $(\mathrm{ExcB} 1 \text { and } \mathrm{ExcB} 4)^{40}$, providing further evidence that subsets of $R O R B$ expressing excitatory neurons in the neocortex are selectively vulnerable.

Considering the Mathys et $a l .{ }^{21}$ and the Marinaro et al. ${ }^{40}$ datasets together with our dataset, it appears that while not all $R O R B$-expressing excitatory neuron subpopulations in the neocortex showed signs of selective vulnerability, those that did were the most similar to RORB-expressing excitatory neurons in the EC, all of which showed signs of selective vulnerability.

\section{Validation of the selective vulnerability of $R O R B$-expressing excitatory neurons}

To validate our finding from snRNA-seq that $R O R B$-expressing excitatory neurons in the EC were highly vulnerable in $\mathrm{AD}$, we performed multiplex immunofluorescence on post-mortem samples from a larger cohort of individuals (Table 1). Specifically, we quantified the proportion of excitatory neurons and RORB-positive excitatory neurons in the EC superficial layers (i.e. above layer IV, which we also refer to as dissecans- $1^{42}$ in Fig. 3b) in postmortem tissue from 26 individuals spanning Braak stage 0 to 6 , who were devoid of non-AD neuropathological changes (Table 1). Given the heterogeneity of the EC, the areas selected for analysis in the caudal EC were delimited using rigorous cytoarchitectonic parameters to minimize the odds of artifactual results (Fig. 3a-c, Extended Data Fig. 6, see Methods). We used multiplex immunofluorescence ${ }^{43}$ to label cells (DAPI), excitatory neurons (TBR1), RORB+ neurons, and phospho-tau neuronal inclusions (CP-13, Ser 202). We failed to find statistically significant changes in the proportion of excitatory neurons overall (TBR1+ cells among all cells) across disease progression (Fig. 3d). However, we observed a substantial reduction in the proportion of RORB+ neurons among excitatory neurons in Braak stages 2-4 and 5-6 compared to Braak stages 0-1 (Fig. 3e).

Furthermore, by analyzing a subset of cases, we detected phospho-tau (CP-13) preferentially in RORB+ compared to RORB- excitatory neurons (Fig. 3f-g). Thus, the above results substantiate that RORB-expressing excitatory neurons are highly vulnerable in $\mathrm{AD}$ and that their depletion parallels the accumulation of tau neurofibrillary inclusions.

Given that large multipolar neurons of "stellate" morphology in EC layer II have been known to be particularly vulnerable in $\mathrm{AD}^{7-10}$, we next examined RORB+ excitatory neurons more closely in terms of morphology by overlaying immunofluorescence with Nissl staining. We found that RORB+ excitatory neurons adopted various shapes, including both pyramidal and multipolar morphologies (Fig. 3h). Conversely, some large multipolar neurons are RORB-negative (Fig. 3h). Thus, our results are consistent with the known vulnerability of large multipolar EC layer II neurons, but also demonstrate that a molecular characterization of vulnerable neurons refines the 318 results of morphological studies.

\section{Lack of differences in vulnerability of inhibitory neuron subpopulations}


Having validated the selective vulnerability of a subpopulation of excitatory neurons, we proceeded to examine inhibitory neurons. It has previously been reported that inhibitory neurons are more resistant to tau pathology compared to excitatory neurons in $\mathrm{AD}^{15,31}$. To investigate whether there are differences among inhibitory neuron subtypes in resilience, we performed subclustering of inhibitory neurons in our dataset, discerning 11 subpopulations in the EC and 10 subpopulations in the SFG (Fig. 4a-d). In both brain regions, inhibitory neuron subpopulations expressed distinct sets of inhibitory neuron subtype markers (Fig. 4a-d), consistent with previous studies $^{19,20}$. We did not any detect statistically significant changes in the relative abundance of inhibitory neurons subpopulations in the EC or SFG (Fig. 4c-d), or in the prefrontal cortex in Mathys et al. ${ }^{21}$ (Extended Data Fig. 7). Although Marinaro et al. reported broad depletion of inhibitory neuron subpopulations in familial monogenic $\mathrm{AD}$, there was no strong evidence of selective vulnerability in particular inhibitory neuron subpopulations relative to other inhibitory neuron subpopulations in Marinaro et al.

\section{Analysis of glial subpopulations}

Glial cells have emerged as important players in AD. We found a trend towards increased relative abundance of microglia in the EC in with $\mathrm{AD}$ progression (Fig. 1f), consistent with microgliosis. Next, we asked whether a specific transcriptional state of microglia is associated with $\mathrm{AD}$ in our dataset. Recent single-cell profiling of microglia from mouse models of $\mathrm{AD}$ identified disease-associated microglia ${ }^{44}$ (DAM), the transcriptional signature of which overlap only partially with that of human microglia found in $\mathrm{AD}^{45}$. Considering the possibility that DAMs may cluster separately from homeostatic microglia after cross-sample alignment, we performed subclustering of microglia in our dataset, discerning 4 subpopulations in the EC and 5 subpopulations in the SFG (Extended Data Fig. 8a-b). However, similar to Thrupp et al. ${ }^{46}$, we were unable to detect the expression of the majority of homeostatic microglia markers and DAM markers in our dataset or in Mathys et al. ${ }^{21}$ (Extended Data Fig. 8d-f), which may be due to the relatively low number of genes captured in microglia compared to other cell types (Fig. 1h-i) and the depletion of many DAM markers in nuclei compared to whole cells ${ }^{46}$.

We next examined oligodendrocytes, which have been shown by Mathys et al. ${ }^{21}$ to exhibit a strong transcriptional response in AD. Subclustering of oligodendrocytes in the EC and SFG revealed subpopulations (EC:Oligo.s0 and EC:Oligo.s4, SFG:Oligo.s1 and SFG:Oligo.s2) which exhibited higher expression of AD-associated oligodendrocyte genes from Mathys et al. ${ }^{21}$, i.e. genes with higher expression in the AD-associated subpopulation Oli0 in Mathys et al. ${ }^{21}$ (Extended Data Fig. 9d-e). Although the function of these genes in the context of AD is largely unknown, a spatial transcriptomics study of $\mathrm{AD}^{47}$ has recently implicated a subset of these genes in the response of oligodendrocytes to amyloid plaques (e.g. $C R Y A B, Q D P R$ ).

Lastly we turned our attention to astrocytes. While reactive astrocytes are ubiquitously associated with $\mathrm{AD}$ pathology ${ }^{48,49}$, only few studies to date have directly profiled reactive astrocytes due to the difficulty of specifically isolating reactive astrocytes ${ }^{50,51}$. Similarly to our interrogation of microglia, we asked if reactive astrocytes would cluster separately from nonreactive astrocytes after cross-sample alignment. After subclustering of astrocytes in our dataset, we discerned 4 subpopulations in the EC and 6 subpopulations in the SFG (Fig. 5a-d). In each 
brain region, there was at least one subpopulation (EC:Astro.3, SFG:Astro.s4 and SFG:Astro.s5) that expressed dramatically higher levels of $G F A P$, which we will refer to as $G F A P_{\text {high }}$ astrocytes (Fig. 5c,d). In the EC, GFAP $P_{\text {high }}$ astrocytes also expressed CD44 and HSPB1, markers of panreactive astrocytes ${ }^{52} ; T N C$, which is upregulated in stab-wound reactive astrocytes ${ }^{53,54}$; and $H S P 90 A A 1$, which is upregulated in reactive astrocytes associated with middle cerebral artery occlusion $^{55}$ (Fig. 5c,d). Interestingly, in the SFG, GFAP ${ }_{\text {high }}$ astrocytes consisted of two subpopulations, one marked by higher expression of $C D 44$ and $T N C$, both of which are involved in interactions with the extracellular matrix, and the other marked by higher expression of HSPB1 and HSP9OAA1, both of which are chaperones involved in proteostasis. In terms of downregulated genes, $G F A P_{\text {high }}$ astrocytes consistently expressed lower levels of genes associated with glutamate/GABA homeostasis (SLC1A2, SLC1A3, GLUL, SLC6A11; see Methods for references) and synaptic adhesion/maintenance (NRXN1, CADM2, PTN, GPC5; see Methods for references), suggesting a loss of homeostatic function.

Examination of all differentially expressed genes in $G F A P_{\text {high }}$ astrocytes compared to other astrocyte subpopulations showed significant overlap with differentially expressed genes from reactive astrocytes in a mouse model of spinal cord injury ${ }^{56}$ (Fig. 5e). Overlapping downregulated genes included the previously noted genes associated with glutamate homeostasis and synaptic adhesion/maintenance and also genes related to lipid metabolism, cytoskeleton and extracellular matrix, and transporters (Fig. 5f-g).

Finally, to confirm the presence of $G F A P_{\text {high }}$ astrocytes in an independent dataset, we performed subclustering of astrocytes from Mathys et $a l .{ }^{21}$ after cross-sample alignment, which yielded 3 subpopulations (Extended Data Fig. 10a,b). Indeed, we found that Mathys:Astro.s2 behaved identically compared to $G F A P_{\text {high }}$ astrocytes from the EC and SFG in terms of upregulating reactive astrocyte markers and downregulating genes associated with glutamate/GABA homeostasis and synaptic adhesion (Extended Data Fig. 10b). Furthermore, the differentially expressed genes in Mathys:Astro.s3 overlapped highly with those in $G F A P_{\text {high }}$ astrocytes from the EC and SFG (Extended Data Fig. 10c).

\section{DISCUSSION}

Selective vulnerability is recognized as a fundamental feature of neurodegenerative diseases, including AD. Past studies have characterized the most vulnerable neurons in AD based on topography and morphology. For instance, EC layer II neurons have been found to be more vulnerable than EC layer III pyramidal neurons ${ }^{10-12}$. However, the molecular signature of selectively vulnerable neurons in AD is largely unknown. In this study, we performed snRNAseq of well-characterized postmortem brain tissue from individuals spanning the progression of AD-type tau neurofibrillary pathology, followed by cross-sample data alignment to identify and characterize selectively vulnerable neuronal populations in the caudal EC and the SFG (Brodmann area 8), representing areas that develop tau neurofibrillary inclusions early and late in the course of $\mathrm{AD}$, respectively. We then validated the snRNA-seq results using quantitative neuropathological methods in a larger cohort spanning all Braak stages of neurofibrillary pathology. 
The transentorhinal region and the EC, hubs for integrating information from hippocampal, cortical and subcortical regions ${ }^{35}$, are the first cortical fields to accumulate tau-positive neurofibrillary inclusions followed by neuronal loss in $\mathrm{AD}^{3}$. The transentorinal region is first affected in Braak stage 1, followed by the EC in Braak stage 2. The EC is a relatively phylogenetically conserved brain structure in mammals ${ }^{35,57}$. The rodent EC can be subdivided into medial and lateral portions based on cytoarchitectonics and projections. In primates, the EC has been subdivided into up to 16 regions that show differential abundances of several neuronal markers, distinct projections, and variation of laminar features ${ }^{11,42,57}$. During evolution, the position of the EC changed, and the mouse medial EC (the source of our layer-specific marker genes) is generally regarded as the equivalent of the caudal EC in humans (our sampling location $)^{36}$. Irrespective of the parcellation scheme adopted, the EC is a heterogeneous structure and cytoarchitectonic considerations matter when analyzing and sampling this region to avoid biased observations ${ }^{42}$.

Neurons in EC layer II are particularly vulnerable in $\mathrm{AD}^{7,9,10,58}$. EC layer II features a mixture of neuronal subpopulations defined by morphology. Large multipolar neurons ("stellate cells"), which are deemed to be excitatory, are abundant ${ }^{59}$. Other neurons assume smaller multipolar morphology and variably sized pyramidal, bipolar, spindle-shaped, and triangular morphologies. Large multipolar neurons are prone to develop AD-tau inclusions and degenerate in very early $\mathrm{AD}$ stages ${ }^{6,60}$. However, variation in the size, shape, and density of these large multipolar neurons along mediolateral and rostrocaudal gradients ${ }^{42,60}$ has hampered rigorous quantitative characterization of their depletion in AD.

Here, we discovered that in the caudal EC, specific excitatory neuron subpopulations defined by snRNA-seq were selectively vulnerable in $\mathrm{AD}$, exhibiting a $\sim 50 \%$ decline in their relative abundance already in early AD stages. These neurons expressed genes associated with layer II of the mouse medial EC, consistent with the known vulnerability of neurons in the superficial layers of the human $\mathrm{EC}$ in $\mathrm{AD}^{7-10}$.

Importantly, we identified $R O R B$ as a marker of these selectively vulnerable excitatory neuron subpopulations, and subsequently validated the selective depletion of RORB+ excitatory neurons in the EC along $\mathrm{AD}$ progression by counting these neurons in a larger cohort of individuals using multiplex immunofluorescence. We found that the selectively vulnerable RORB+ excitatory neurons included both large multipolar neurons and pyramidal neurons. Although this finding is consistent with the known vulnerability of large multipolar EC layer II neurons, it also demonstrates that morphology alone is insufficient to determine gradients of selective vulnerability.

We then showed that tau neuronal inclusions, a chief AD neuropathological hallmark, preferentially accumulated in RORB+ excitatory neurons in the EC. To uncover potential cell biological mechanisms underlying the vulnerability of EC RORB+ excitatory neurons, we compared the gene expression profiles of EC RORB-expressing excitatory neurons against all other EC excitatory neurons, which revealed differences in the expression of genes encoding synapse- vs. axon-localized proteins, potassium channel subunits, G-protein signaling molecules, and neurotransmitter receptor signaling molecules. Future studies utilizing in vitro and animal models of $\mathrm{AD}$ together with techniques for manipulating gene expression such as CRISPR 
inhibition and activation ${ }^{61-63}$ will make it possible to address these potential mechanistic connections among RORB-expression, phospho-tau accumulation, and vulnerability.

In neocortical areas, layers III and $\mathrm{V}$ are the first to accumulate tau neurofibrillary inclusions in $\mathrm{AD}^{3,32,33}$. We found that in the SFG, a subset of $R O R B$-expressing excitatory neuron subpopulations showed signs of selective vulnerability only late in $\mathrm{AD}$, in line with the late appearance of neurofibrillary inclusions in the SFG, although the decrease in their relative abundance did not pass our threshold for statistical significance after correction for multiple testing. Interestingly, we found through correlation analysis of gene expression and also EC-SFG cross-sample alignment that $R O R B$-expressing excitatory neuron subpopulations in the SFG showing signs of selective vulnerability were similar to those in the EC in terms of their transcriptomic profile. To verify the reproducibility of our findings, we re-analyzed the data from Mathys et al..$^{21}$ using our cross-sample alignment approach. Although Mathys et al. ${ }^{21}$ probed a different neocortical region (the prefrontal cortex), we found that one of their RORB-expressing excitatory neuron subpopulations also exhibited selective vulnerability and mapped to our $R O R B$-expressing excitatory neuron subpopulations in the EC. In addition, Marinaro et al. ${ }^{40}$, which examined familial monogenic AD, also reported the selective depletion of two out four $R O R B$-expressing excitatory neuron subpopulations in their dataset. Considering our dataset jointly with the Mathys et $a .^{21}$ and Marinaro et $a .^{40}$ datasets, it appears that in the neocortex, while not all $R O R B$-expressing excitatory neuron subpopulations are selectively vulnerable, those that are vulnerable have a similar transcriptional profile as selectively vulnerable neurons in the EC. Given that RORB is known to function as a developmental driver of neuronal subtype identity in the neocortex ${ }^{37-39}$, we hypothesize that the vulnerability of $R O R B$-expressing excitatory neuron subpopulations in different brain regions is caused by the activity of RORB and potentially other subtype-determining transcription factors, which drive gene expression programs that confer an intrinsic vulnerability of these neurons that is realized in the presence of AD-tau pathology. Further mechanistic studies involving the perturbation of $R O R B$ expression in animal models of $\mathrm{AD}$ are necessary to test this hypothesis.

A previous study suggested changes in the number of neurons expressing calbindin and parvalbumin, which tend to mark inhibitory neurons, in EC layer II in $\mathrm{AD}^{58}$. Here, we found no evidence of selective vulnerability in inhibitory neurons subpopulations in EC layer II or any other layer. Inhibitory neurons in the EC superficial layers show a gradient of abundance in the various EC regions ${ }^{35}$, which could confound the results. But, given that we used strict cytoarchitectonic criteria to sample the EC, it is unlikely that our results reflect comparisons of different EC areas across the cases. Also, evidence suggest that these inhibitory neurons undergo changes in morphology and function, rather than loss in sporadic $\mathrm{AD}^{58}$. Thus, our results do not preclude the possibility that inhibitory neuron subpopulations may be differentially affected by $\mathrm{AD}$ progression at the morphological and likely functional level, even if neuronal loss is not apparent.

Until recently, $\mathrm{AD}$ research was mostly neuron-centric, but accumulating evidence is highlighting the importance of glial changes in the pathogenesis of $\mathrm{AD}$. Although we could not detect the disease-associated microglia signature ${ }^{44,45}$ in our study, likely due the low number of transcripts recovered in microglia, we discovered an astrocyte subpopulation expressing high levels of GFAP, which we termed $G F A P_{\text {high }}$ astrocytes, in both the EC and SFG, as well as in the 
prefrontal cortex from Mathys et al. ${ }^{21} \mathrm{We}$ found that $G F A P_{\text {high }}$ astrocytes also expressed higher levels of other genes associated with reactive astrocytes, while expressing lower levels of genes involved in glutamate homeostasis and synaptic adhesion/maintenance, which suggests loss of normal astrocyte homeostatic functions. Furthermore, we found a high degree of overlap between genes differentially expressed in $G F A P_{\text {high }}$ astrocytes and genes differentially expressed in reactive astrocytes from a mouse model of spinal injury ${ }^{56}$. Thus, we believe that $G F A P_{\text {high }}$ astrocytes correspond to reactive astrocytes in $\mathrm{AD}$, which may have compromised homeostatic function.

Our study has several methodological strengths. First, the postmortem cohort used for snRNAseq and histopathological validation consists of well-characterized cases, devoid of non-AD pathology. To minimize confounders in the snRNA-seq results, we selected only male cases with an $A P O E$ \&3/\&3 genotype. Second, we sequenced a very large number of nuclei from each case $\left(\sim 10,000\right.$ nuclei per case, compared to $\sim 1,700$ nuclei per case in Mathys et al $\left.{ }^{21}\right)$ from two brain regions per individual ( 4,000 nuclei from the EC and $\sim 6,000$ nuclei from the SFG). Third, the human cortex displays a complex parcellation scheme based on cytoarchitectonic characteristics that reflect differences in the abundance of various cell subpopulations, with implications for function, projections, and differential vulnerability in AD. Many RNA-seq studies of AD used broad descriptions to define the sampled brain areas, making it challenging to understand if they were sampled from the same subfields. We used strict cytoarchitectonic criteria to sample brain regions for snRNA-seq and histopathological validation. Fourth, our focus was on defining cell type subpopulations that showed changes in relative abundance between disease stages, which can reflect important disease processes such as neuronal loss, and to define the genes characteristic of these subpopulations. The way we defined cell type subpopulations independently of disease progression allowed us to compare gene expression between different cell type subpopulations within individuals while controlling for differences among individuals; this is more robust than comparing gene expression in a given subpopulation across groups of individuals, which can be influenced by differences in confounding factors between the groups. Lastly, by validating our findings using a novel multiplex immunofluorescence approach that enables probing a higher number of antibodies simultaneously ${ }^{43}$, we could quantify the relative abundance of excitatory neurons and RORB+ neurons and also demonstrate that RORB+ excitatory neurons were preferentially affected by neurofibrillary inclusions.

A limitation of our study is that we only included male $A P O E \varepsilon 3 / \varepsilon 3$ individuals in the snRNAseq analysis. We included females and individuals carrying the APOE $\varepsilon 4$ allele associated with AD risk in our histopathological validation, but caution should be taken before generalizing our results to these groups. Future studies will provide a systematic analysis of the impact of sex and $A P O E$ status on selective vulnerability in AD.

In conclusion, our study contributes, to the best of our knowledge, a pioneering characterization of selectively vulnerable neuronal populations in AD using snRNA-seq profiling of paired brain regions from the same individuals, which were all carefully curated $\mathrm{AD}$ cases and controls. These results will inform future studies of the mechanistic basis of selective vulnerability in both animal and in vitro models, such as human iPSC-derived neurons, in which the deployment of CRISPR inhibition and activation technology enables elucidation of the functional consequences of transcriptomic changes ${ }^{61,64}$. 
bioRxiv preprint doi: https://doi.org/10.1101/2020.04.04.025825; this version posted August 23, 2020. The copyright holder for this preprint (which was not certified by peer review) is the author/funder. All rights reserved. No reuse allowed without permission. 


\section{ONLINE METHODS}

\section{Post-mortem cohort}

This study was approved by and University of Sao Paulo institutional review board and deemed non-human subject research by the University of California, San Francisco (UCSF). Deidentified human postmortem brain tissue was supplied by the Neurodegenerative Disease Brain Bank (NDBB) at UCSF, and the Brazilian BioBank for Aging Studies (BBAS) from the University of Sao Paulo ${ }^{65}$. The NDBB receives brain donations from patients enrolled in the UCSF Memory and Aging Center research programs. The BBAS is population $\square$ based and houses a high percentage of pathologically and clinically normal control subjects who are not available in the NDBB. Neuropathological assessments were performed using standardized protocols and followed internationally accepted criteria for neurodegenerative diseases ${ }^{66-68}$. The brain samples used in this study contained a broad burden of AD-type pathology and were selected to be free from non-AD pathology including Lewy body disease, TDP-43 proteinopathies, primary tauopathies, and cerebrovascular changes. Argyrophilic grain disease (AGD) was not an exclusion criterion based on its high prevalence and lack of correlation with significant clinical symptoms ${ }^{69-71}$. In total, the cohort included 10 cases who underwent snRNAseq, representing Braak stages 0, 2 and 6, all ApoE 3/3, and 26 cases who underwent neuroanatomical analysis, representing Braak stages $0-6^{3,25}$, ranging from 2-5 individuals per Braak stage. Table 1 depicts the characteristics of the 31 cases.

\section{Isolation of nuclei from frozen post-mortem human brain tissue}

Isolation of nuclei was performed similarly as previously described ${ }^{72}$. Briefly, frozen brain tissue was dounce homogenized in $5 \mathrm{ml}$ of lysis buffer $(0.25 \mathrm{M}$ sucrose, $25 \mathrm{mM} \mathrm{KCl}, 5 \mathrm{mM} \mathrm{MgCl}, 20$ $\mathrm{mM}$ Tricine-KOH, pH 7.8, $1 \mathrm{mM}$ DTT, $0.15 \mathrm{mM}$ spermine, $0.5 \mathrm{mM}$ spermidine, $1 \mathrm{X}$ protease inhibitor (Sigma, 4693159001), and RNAse Inhibitor (Promega, N2615)). Following initial dounce homogenization, IGEPAL-630 was added to a final concentration of $0.3 \%$ and the sample was homogenized with 5 more strokes. The solution was then filtered through a 40 um cell filter and mixed with Optiprep (Sigma, D1556-250ML) to create a 25\% Optiprep solution. This solution was then layered onto a 30\%/40\% Optiprep gradient and centrifuged at 10,000g for 18 minutes using the SW41-Ti rotor. The nuclei were collected at the 30\%/40\% Optiprep interface.

\section{Droplet-based single-nucleus RNA-sequencing}

Droplet-based single-nucleus RNA-sequencing (snRNA-seq) was performed using the Chromium Single Cell 3' Reagent Kits v2 from 10X Genomics. Nuclei were resuspended to a concentration of 1000 nuclei/uL in $30 \%$ Optiprep solution before loading according to manufacturer's protocol, with 10,000 nuclei recovered per sample as the target. cDNA fragment analysis was performed using the Agilent 4200 TapeStation System. Sequencing parameters and quality control were performed as described by The Tabula Muris Consortium ${ }^{73}$.

\section{Pre-processing of snRNA-seq data}

Sequencing data generated from snRNA-seq libraries were demultiplexed using Cellranger

600 GRCh38 reference genome using cellranger mkref in order to account for introns that may be 
601

602

603

604

605

606

607

608

609

610

611

612

613

614

615

616

617

618

619

620

621

622

623

624

625

626

627

628

629

630

631

632

633

634

635

636

637

638

639

640

641

642

643

644

645

646

eliminated using the default GRCh38 reference genome. Alignment and gene expression quantification was then performed using cellranger count with default settings.

\section{Exploratory analysis of EC and SFG data}

For each sample, the raw gene-barcode matrix outputted by Cellranger (version 2.1.0) was converted into a SingleCellExperiment (SCE) object using the read10xCounts function from the DropletUtils package ${ }^{74}$ (version 1.2.2). Droplets containing nuclei were then distinguished from empty droplets using DropletUtils::emptyDrops with the parameter $F D R=0.01$, and then nuclei (hereon also referred to as "cells") with less than 200 UMIs were discarded. Afterwards, SCE objects corresponding to each sample were merged into a single SCE object for downstream processing and analyses.

For normalization of raw counts, to avoid artifacts caused by data sparsity, the approach of Lun et al. ${ }^{75}$ was adopted: For each sample, cells were first clustered using a graph-based method followed by pooling counts across cells in each cluster to obtain pool-based size factors, which were then deconvoluted to yield cell-based size factors. Clustering was performed using the quickCluster function from the scran package ${ }^{76}$ (version 1.10.2) with the parameters method $=$ 'igraph', min.mean $=0.1$, irlba.args $=$ list $($ maxit $=1000)$, and the block parameter set to a character vector containing the sample identity of each cell. Size factors were computed using scran:: computeSumFactors with the parameter min.mean $=0.1$ and the cluster parameter set to a character vector containing the cluster identity of each cell; cells with negative size factors were removed. Normalization followed by log-transformation was then performed using the normalize function from the scater package ${ }^{77}$ (version 1.10.1).

Prior to dimensionality reduction, highly variable genes were identified for each sample separately using the approach of Lun et $a .^{76}$ : Each gene's variance was decomposed into a technical and biological component. Technical variance was assumed as Poisson and modeled using scran::makeTechTrend. The mean-variance trend across genes was fitted using scran::trendVar with parameters use.spikes $=F A L S E$ and loess.args $=l i s t($ span $=0.05)$; and the trend slot of the resulting fit object was then set to the output of scran::makeTechTrend.

Biological variance was extracted from the total variance using scran::decomposeVar with the above fit object as the input. Finally, highly variable genes that were preserved across samples were identified by combining the variance decompositions with scran::combineVar, using Stouffer's z-score method for meta-analysis ( $\operatorname{method}=$ ' $z$ '), which assigns more weight to samples with more cells.

For initial data exploration, genes with combined biological variance greater than 0 were used as the feature set for dimensionality reduction by principal component analysis using scran::parallelPCA, which uses Horn's parallel analysis to decide how many principal components to retain, with parameter approx $=$ TRUE. Clustering was then performed on the retained principal components using the FindClusters function from the Seurat package ${ }^{78}$ (version 2.3.4) with parameter resolution $=0.8$, which required conversion of SCE objects to Seurat objects using Seurat $:$ Convert. To visualize the clusters, t-stochastic neighborhood embedding (tSNE) was performed on the retained principal components using scater::runTSNE with parameters perplexity $=30$ and rand_seed $=100$. 


\section{Cross-sample alignment of SFG and EC data}

648 Initial data exploration revealed that clustering was driven by individual of origin in addition to cell type identity, which makes it difficult to analyze changes in the relative abundance or gene expression of a given cell type across disease progression or brain regions. To recover clusters defined by mainly by cell type identity, data was aligned across samples from each brain region using with $s c$ Align $^{29}$ (version 1.0.0), which leverages a neural network to learn a lowdimensional alignment space in which cells from different datasets group by biological function independent of technical and experimental factors. As noted by Johansen \& Quon ${ }^{29}$, scAlign converges faster with little loss of performance when the input data is represented by principal components or canonical correlation vectors. Therefore, prior to running scAlign, the top 2000 genes with the highest combined biological variance were used as the feature set for canonical correlation analysis (CCA), which was implemented using Seurat::RunMultiCCA with parameter num.cc $=15$. The number of canonical coordinates to use for scAlign was determined by the elbow method using Seurat::MetageneBicorPlot. scAlign was then run on the cell loadings along the top 10 canonical correlation vectors with the parameters options $=$ scAlignOptions $($ steps $=$ 10000 , log.every $=5000$, architecture $=$ 'large', num.dim $=64)$, encoder.data $=$ 'cca', supervised $=$ 'none', run.encoder $=$ TRUE, run.decoder $=$ FALSE, log.results $=$ TRUE, and device $=$ ' $C P U$ '. Clustering was then performed on the full dimensionality of the ouptut from scAlign using Seurat::FindClusters with parameter resolution $=0.8$ for the SFG and resolution $=0.6$ for the EC. Clusters were visualized with tSNE using Seurat::RunTSNE on the full dimensinality of the output from scAlign with parameter do.fast $=$ TRUE. Alignment using scAlign followed by clustering was also performed for all samples from both brain regions jointly.

To assign clusters identified in the aligned subspace generated by scAlign to major brain cell types, the following marker genes were used: $S L C 17 A 7$ and $C A M K 2 A$ for excitatory neurons, $G A D 1$ and $G A D 2$ for inhibitory neurons, SLC1A2 and $A Q P 4$ for astrocytes, $M B P$ and $M O G$ for oligodendrocytes, PDGFRA and SOX10 for oligodendrocyte precursor cells (OPCs), CD74 and CX3CR1 for microglia/myeloid cells, and CLDN5 and FLT1 for endothelial cells. Clusters expressing markers for more than one cell type, most likely reflecting doublets, were removed from downstream analyses.

\section{Cell type-specific subclustering (subpopulation) analysis}

To identify cell type subpopulations, cells from all samples belonging to a given major cell type were extracted for sample-level re-computation of size factors and highly variable genes. CCA was then performed using the top 1000 genes with the highest combined biological variance as the feature set, followed by alignment of the first 10 to 12 canoical coordinates with scAlign, with steps $=2500$. The full dimensionality of the output from scAlign was used for subclustering (using resolution $=0.4$ ) and tSNE. Analyzing cells from each brain region separately, marker genes for subpopulations were identified using scran::findMarkers with parameters direction = 'up', pval.type $=$ 'any', lfc $=0.58$, and the block parameter set to a character vector corresponding to each cell's sample identity. Subpopulations that expressed markers for more than one cell type were removed from downstream analyses.

690

691 Identification of differentially expressed genes in cell type subpopulations 
To identify genes differentially expressed by a cell type subpopulation compared to all other subpopulations in a way that accounts for true biological replication (i.e. at the level of individuals), UMI counts of cells from the same individual belonging to the subpopulation of interest or all other subpopulations were summed to obtain "pseudo-bulk" samples, which were then analyzed using $e d g e R^{79}$ (version 3.24.3) following the approach recommended by Amezquita $e t a l .{ }^{80}$ A false-discovery rate cutoff of 0.1 was used.

\section{Heatmap visualization of relative gene expression across cell types or cell type subpopulations} the figures, $\log$-scaled normalized counts of each gene were z-score transformed across all cells and then averaged across cells in each cluster to enhance visualization of differences among clusters. Thus genes with "high" relative expression have above-average expression (positive zscores) and genes with "low" relative expression have below-average expression (negative zscores).

\section{Functional association network analysis and pathway enrichment analysis of differentially expressed genes}

Differentially expressed genes were visualized as a functional association network using String$\mathrm{db}^{81}$ (v11), a protein-protein association network based on known physical interactions, functional associations, coexpression, and other metrics, and Cytoscape ${ }^{82}$ (version 3.7.2), a network visualization software. When generating the networks, the String-db association confidence score cutoff set to 0.5 , and the network layout was optimized for visualization using the yFiles Organic Layout. For pathway enrichment analysis, enrichments for Gene Ontology terms and Reactome Pathways were also obtained through String-db, using a false-discovery rate cutoff of 0.05 .

\section{Beta regression}

For each brain region, to determine the statistical significance of changes in the relative abundance of a given cluster or cell type across disease progression, the relative abundance was computed for each sample and treated as an independent measurement and beta regression ${ }^{30}$ was performed using the betareg package ${ }^{83}$ (version 3.1-1), using the formula relative.abundance braak.stage for both the mean and precision models, and the bias-corrected maximum likelihood estimator (type $=$ ' $B C$ '). The statistical significance of changes in the proportion of TBR $1+$ cells and RORB+ cells among TBR1+ cells obtained from immunofluorescence validation were assessed similarly as above using beta regression. To correct for multiple hypothesis testing for each family of tests (e.g. testing all cell type subpopulations for a brain region), Holm's method was used to adjust $P$ values obtained from beta regression to control the family-wise type I error rate at 0.05 .

\section{Entorhinal cortex layer-specific genes}

Due to the lack of published data on layer-specific genes for the human EC, layer-specific genes in the mouse medial entorhinal cortex (MEC) were obtained from Ramsden et al. ${ }^{34}$. (The MEC is the most phylogenetically similar to the human caudal EC ${ }^{35,36}$ used in this study.) Specifically, genes with expression specific for layer II, III, and V/VI of the mouse MEC according to the S4 Dataset excel spreadsheet in the supplemental information of Ramsden et al. ${ }^{34}$ were mapped to 
human genes, and cross-referenced against genes differentially expressed across EC excitatory neuron subclusters (obtained using scran::findMarkers without setting direction = 'up').

\section{Re-analysis of the Mathys et al. dataset}

To re-analyze the data from Mathys $e t a .^{21}$ using our cross-sample alignment approach, the filtered matrix of UMI counts ("filtered_count_matrix.mtx") and associated row and column metadata were downloaded from The AMP-AD Knowledge Portal (Synapse ID: syn18485175). Since clinical metadata was not provided in the column metadata, the individual ID ("projid" column in the column metadata) was cross-referenced with the official ROS-MAP clinical metadata ("ROSMAP_Clinical_2019-05_v3.csv", synapse ID: syn3191087), which was then cross-referenced with the additional metadata provided in Supplementary Table 1 and 3 from Mathys $e t a l .{ }^{21}$ The filtered UMI counts matrix and the associated row and column metadata were then converted to a SingleCellExperiment object for analysis. The cell type assignments from Mathys et al. ${ }^{21}$ provided in the column metadata were used for subclustering.

\section{Functional annotation of differentially expressed genes in $G F A P_{\text {high }}$ astrocytes}

We obtained the functional annotation for differentially expressed genes from the GeneCards website $^{84}$ and verified the primary literature references for glutamate/GABA-related genes ${ }^{85-90}$ and synaptic adhesion/maintenance-related genes ${ }^{91-94}$.

\section{Quantitative histopathological assessment using multiplex immunofluorescence}

Delineation of the caudal EC. We used archival paraffin blocks from the UCSF/NBDD and BBAS (Table 1). First, we collected blocks sampling the hippocampal formation anterior to the lateral genicular body from the 10 cases used for the snRNAseq and another 30 cases spanning all Braak stages ${ }^{3}$. To determine if the caudal EC region was present, $8 \mu \mathrm{m}$ thick sections of each block underwent hematoxylin and eosin staining (Extended Data Fig. 8A). We took digital images of the stained sections and aligned each one the most approximate section from a large collection of $400 \mu \mathrm{m}$ thick serial coronal sections of whole-brain hemispheres stained for gallocyanin provided by co-author Heinsen ${ }^{42,95}$ (Extended Fig Data 8B). We eliminated blocks from five cases used for snRNA-seq and four of the extra cases for lack of caudal EC. Next, again with the aid of the paired gallocyanin sections, we delineated the borders of the caudal EC in each case (Extended Data Fig. 8A).

The EC is considered a peri- or allocortex, depending on the author ${ }^{11}$. EC parcellation and cytoarchitectonic definitions have been a matter of debate, and here, we are adopting the cytoarchitectonic definitions proposed by Heinsen and colleagues ${ }^{42}$, which is based on the examination of thick histological preparations and considered the definitions proposed by Insausti and Amaral (6 layers) ${ }^{96}$ and Braak and Braak (3 layers) $)^{11}$. In thick histological sections, the caudal entorhinal region features well-delineated clusters of stellate or principal cells in layer II (pre-alpha clusters) and three lamina dissecans ${ }^{42}$. The external dissecans (dissecans-ext) divides layers II and III is particularly prominent in the caudal EC. Dissecans-1 (diss-1) corresponds to layer IV of Insausti ${ }^{57}$ and the lamina dissecans of Braak and Braak ${ }^{11}$ and Rose ${ }^{97}$. The most internal dissecans (dissecans-2, or diss-2) is hardly appreciated in thin sections but easy to visualize in thick sections. It roughly corresponds to layer Vc of the caudal subregions of Insausti $^{57}$. 
Multiplex immunofluorescence. Next, for each case, an $8 \mu \mathrm{m}$ thick, formalin-fixed and paraffinembedded coronal section underwent immunofluorescence against TBR1, RORB and phosphotau(CP-13) as described below. TBR1, or T-box, brain, 1 is a transcription factor protein that has a role in differentiation of glutamatergic neurons and is a marker for excitatory neurons, including EC excitatory neurons ${ }^{14,98}$. In summary, sections were deparaffinized and incubated in $3.0 \%$ hydrogen peroxide (Fisher, H325-500) in methanol to inactivate endogenous peroxidase. Antigen retrieval was performed in 1X Tris-EDTA HIER solution (TES500) PBS with $0.05 \%$ Tween 20 (PBS-T) at pH9 in an autoclave at $121 \square^{\circ} \mathrm{C}$ for five $\square$ minutes. To reduce nonspecific background staining, sections were blocked with 5\% Milk/PBS-T. To avoid cross-reactions between primary antibodies that were raised against the same species, an antibody stripping step using $0.80 \% \beta$-mercaptoethanol/10\% sodium dodecyl sulfate in $12.5 \%$ Tris-HCL was performed after the tyramide-signal amplification (TSA) development for RORB.

Sections were first incubated overnight in primary antibody against RORB (1:400, rabbit, HPA008393, Millipore Sigma), which was later developed in goat anti-rabbit HRP (1:400, R05072-500, Advansta) with Alexa Fluor 488 TSA (1:100, B40953, Thermo Fisher). Next, sections were stripped of RORB primary antibody and then were incubated overnight in a cocktail of primary antibodies against TBR1 (1:100, Rabbit, ab31940, Abcam) and CP13 (1:800, mouse, phospho-tau serine 202, gift of Peter Davies, NY), all of which were later developed with secondary antibodies and fluorophores: for TBR1, Alexa Fluor 546 conjugated anti-rabbit secondary (1:200, A-11010, Thermo Fisher) was used, and for CP13, biotinylated anti-mouse (1:400, BA-2000, Vector Laboratory) with streptavidin Alexa Fluor 790 (1:250, S11378, Thermo Fisher) was used. Sections were then counterstained with DAPI diluted in PBS (1:5000, D1306, Invitrogen). Finally, sections were then incubated in Sudan Black B (199664-25g, Sigma) to reduce autofluorescence and coverslipped using Prolong antifade mounting media (P36980, Invitrogen). A quality control slide was used to verify the efficacy of the antibody stripping process. A detailed description of the method is provided in Ehrenberg et al. ${ }^{43}$ Sections were scanned using a Zeiss AxioScan Slide Scanner.

For generating the images shown in Fig. 3h, a section from case \#6 (Braak stage 2, see Table 1) was stained with gallocyanin-chrome alum following standard methods ${ }^{42}$. The section was placed on a cover slip and scanned using a Zeiss AxioScan Slide Scanner. Next, the section was removed from the cover slip and underwent immunofluorescence for RORB and CP13 as described above. Then, the section was placed on a cover slip and scanned once more.

Neuronal quantification. The caudal EC delineations carried out in the hematoxylin and eosinstained slides were then transferred to the immunostained images. Within these borders, we randomly placed four 500x500 $\mu \mathrm{m}$ regions of interest (ROI) overlaying the EC external layers (I to III), which we identified as being external to dissecans-1. We then extracted the ROIs for quantification in ImageJ (Fig. 3). The number of excitatory neurons was quantified by segmenting the TBR1 signal, using a threshold to create a mask and the segmentation editor plugin to manually remove all non-neuronal artifacts and vessels. The number of RORB+ excitatory neurons was then counted using the mask of excitatory (TBR1+) neurons in the segmentation editor and manually removing all neurons not expressing RORB. All 
segmentations were manually verified for quality control. Quantification was done blinded to the neuropathological diagnosis. We quantified phospho-tau (CP-13) staining in two ROIs in a subset of the cases, using the same FIJI protocol.

\section{DATA AVAILABILITY}

The raw snRNA-seq sequencing data and unfiltered UMI count matrices are available on the Gene Expression Omnibus (GEO) under the accession GSE147528. Single-cell data after quality control is available for download in synapse.org at under the Synapse ID syn21788402. Post quality-control data can also be explored interactively through the CellXGene platform at https://kampmannlab.ucsf.edu/ad-brain.

\section{CODE AVAILABILITY}

We provide the full bioinformatics pipeline for the analysis of snRNA-seq data in this paper at https://kampmannlab.ucsf.edu/ad-brain-analysis.

\section{ACKNOWLEDGEMENTS}

We thank Angela Pisco, Ashley Maynard, Spyros Darmanis and MACA team at the Chan Zuckerberg Biohub for advice on analysis 10X library preparation and reagents. We thank members of the Kampmann lab (Avi Samelson, Xiaoyan Guo, Ruilin Tian, Brendan Rooney) for feedback on the manuscript. This work was supported by NIH awards F30 AG066418 (K.L.), K08 AG052648 (S.S.), R56 AG057528 (M.K., L.T.G.), K24 AG053435 (L.T.G), U54 NS100717 (L.T.G, M.K.), an NDSEG fellowship (E.L.), Alzheimer's Association fellowship AARF 18-566005 (R.D.R.), FAPESP/CAPES (2016/24326-0) (R.D.R.) and a Chan Zuckerberg Biohub Investigator Award (M.K.). The UCSF Neurodegenerative Disease Brain Bank is supported by NIH grants AG023501 and AG019724, the Tau Consortium, and the Bluefield Project to Cure FTD.

\section{AUTHOR CONTRIBUTIONS}

K.L., E.L., L.T.G. and M.K. conceptualized and led the overall project. K.L., L.T.G. and M.K. wrote the manuscript, with input from all co-authors. K.L. analyzed snRNA-Seq data and visualized results. E.L. generated snRNA-Seq data, with support from R.S., M.T., and N.N. generation and analysis, R.E., A.P. and H.H. contributed to neuropathological data analysis, and S.H.L. contributed to neuropathological method development.

\section{COMPETING INTERESTS}

The authors declare no competing interests.

\section{ETHICS DECLARATIONS}

This project was approved the the ethical committee of the University of Sao Paulo (for tissue transfer) and deemed non-human subject research by UCSF. 


\section{REFERENCES}

1. Fu, H., Hardy, J. \& Duff, K.E. Selective vulnerability in neurodegenerative diseases. Nat Neurosci 21, 1350-1358 (2018).

882 2. Saxena, S. \& Caroni, P. Selective neuronal vulnerability in neurodegenerative diseases: 883 from stressor thresholds to degeneration. Neuron 71, 35-48 (2011).

884 3. Braak, H. \& Braak, E. Neuropathological stageing of Alzheimer-related changes. Acta 885 Neuropathol 82, 239-259 (1991).

886 4. Scholl, M., et al. PET Imaging of Tau Deposition in the Aging Human Brain. Neuron 89, 887 971-982 (2016).

888 5. Seeley, W.W., Crawford, R.K., Zhou, J., Miller, B.L. \& Greicius, M.D.

889 Neurodegenerative diseases target large-scale human brain networks. Neuron 62, 42-52 (2009).

890 6. Braak, H. \& Braak, E. Staging of Alzheimer's disease-related neurofibrillary changes.

891 Neurobiol Aging 16, 271-278; discussion 278-284 (1995).

$8927 . \quad$ Price, J.L., et al. Neuron number in the entorhinal cortex and CA1 in preclinical 893 Alzheimer disease. Arch Neurol 58, 1395-1402 (2001).

894 8. Stranahan, A.M. \& Mattson, M.P. Selective vulnerability of neurons in layer II of the 895 entorhinal cortex during aging and Alzheimer's disease. Neural Plast 2010, 108190 (2010).

$8969 . \quad$ Van Hoesen, G.W., Hyman, B.T. \& Damasio, A.R. Entorhinal cortex pathology in 897 Alzheimer's disease. Hippocampus 1, 1-8 (1991).

898 10. Gomez-Isla, T., et al. Profound loss of layer II entorhinal cortex neurons occurs in very 899 mild Alzheimer's disease. J Neurosci 16, 4491-4500 (1996).

900 11. Braak, H. \& Braak, E. The human entorhinal cortex: normal morphology and lamina901 specific pathology in various diseases. Neurosci Res 15, 6-31 (1992).

902 12. Kordower, J.H., et al. Loss and atrophy of layer II entorhinal cortex neurons in elderly 903 people with mild cognitive impairment. Ann Neurol 49, 202-213 (2001).

904 13. Gotz, J., Schonrock, N., Vissel, B. \& Ittner, L.M. Alzheimer's disease selective 905 vulnerability and modeling in transgenic mice. Journal of Alzheimer's disease : JAD 18, 243-251 906 (2009).

907 14. Fu, H., et al. Tau Pathology Induces Excitatory Neuron Loss, Grid Cell Dysfunction, and 908 Spatial Memory Deficits Reminiscent of Early Alzheimer's Disease. Neuron 93, 533-541 e535 909 (2017). 
910 15. Fu, H., et al. A tau homeostasis signature is linked with the cellular and regional 911 vulnerability of excitatory neurons to tau pathology. Nature neuroscience 22, 47-56 (2019).

912 16. Drummond, E. \& Wisniewski, T. Alzheimer's disease: experimental models and reality. 913 Acta Neuropathol 133, 155-175 (2017).

914 17. Liang, W.S., et al. Altered neuronal gene expression in brain regions differentially 915 affected by Alzheimer's disease: a reference data set. Physiol Genomics 33, 240-256 (2008).

916 18. Liang, W.S., et al. Gene expression profiles in anatomically and functionally distinct 917 regions of the normal aged human brain. Physiol Genomics 28, 311-322 (2007).

918 19. Lake, B.B., et al. Neuronal subtypes and diversity revealed by single-nucleus RNA 919 sequencing of the human brain. Science 352, 1586-1590 (2016).

920 20. Hodge, R.D., et al. Conserved cell types with divergent features in human versus mouse 921 cortex. Nature 573, 61-68 (2019).

922 21. Mathys, H., et al. Single-cell transcriptomic analysis of Alzheimer's disease. Nature 570, 923 332-337 (2019).

924 22. Grubman, A., et al. A single-cell atlas of entorhinal cortex from individuals with 925 Alzheimer's disease reveals cell-type-specific gene expression regulation. Nature neuroscience 926 22, 2087-2097 (2019).

927 23. Mirra, S.S., et al. The Consortium to Establish a Registry for Alzheimer's Disease 928 (CERAD). Part II. Standardization of the neuropathologic assessment of Alzheimer's disease. 929 Neurology 41, 479-486 (1991).

930 24. Thal, D.R., Rub, U., Orantes, M. \& Braak, H. Phases of A beta-deposition in the human 931 brain and its relevance for the development of AD. Neurology 58, 1791-1800 (2002).

$932 \quad 25 . \quad$ Braak, H., Thal, D.R., Ghebremedhin, E. \& Del Tredici, K. Stages of the pathologic 933 process in Alzheimer disease: age categories from 1 to 100 years. J Neuropathol Exp Neurol 70, 934 960-969 (2011).

935 26. Nelson, P.T., et al. Correlation of Alzheimer disease neuropathologic changes with 936 cognitive status: a review of the literature. J Neuropathol Exp Neurol 71, 362-381 (2012).

937 27. Butler, A., Hoffman, P., Smibert, P., Papalexi, E. \& Satija, R. Integrating single-cell 938 transcriptomic data across different conditions, technologies, and species. Nat Biotechnol 36, 939 411-420 (2018).

940 28. Haghverdi, L., Lun, A.T.L., Morgan, M.D. \& Marioni, J.C. Batch effects in single-cell 941 RNA-sequencing data are corrected by matching mutual nearest neighbors. Nat Biotechnol 36, 942 421-427 (2018). 
943 29. Johansen, N. \& Quon, G. scAlign: a tool for alignment, integration, and rare cell 944 identification from scRNA-seq data. Genome biology 20, 166 (2019).

945 30. Ferrari, S.L.P. \& Cribari-Neto, F. Beta regression for modelling rates and proportions. $J$ 946 Appl Stat 31, 799-815 (2004).

947 31. Hof, P.R., et al. Parvalbumin-immunoreactive neurons in the neocortex are resistant to 948 degeneration in Alzheimer's disease. J Neuropathol Exp Neurol 50, 451-462 (1991).

949 32. Hof, P.R., Cox, K. \& Morrison, J.H. Quantitative analysis of a vulnerable subset of 950 pyramidal neurons in Alzheimer's disease: I. Superior frontal and inferior temporal cortex. $J$ 951 Comp Neurol 301, 44-54 (1990).

952 33. Hof, P.R. \& Morrison, J.H. Neocortical neuronal subpopulations labeled by a monoclonal 953 antibody to calbindin exhibit differential vulnerability in Alzheimer's disease. Exp Neurol 111, 954 293-301 (1991).

955 34. Ramsden, H.L., Surmeli, G., McDonagh, S.G. \& Nolan, M.F. Laminar and dorsoventral 956 molecular organization of the medial entorhinal cortex revealed by large-scale anatomical 957 analysis of gene expression. PLoS Comput Biol 11, e1004032 (2015).

958 35. Kobro-Flatmoen, A. \& Witter, M.P. Neuronal chemo-architecture of the entorhinal 959 cortex: A comparative review. Eur J Neurosci 50, 3627-3662 (2019).

960 36. Naumann, R.K., et al. Conserved size and periodicity of pyramidal patches in layer 2 of 961 medial/caudal entorhinal cortex. J Comp Neurol 524, 783-806 (2016).

$962 \quad 37 . \quad$ Jabaudon, D., Shnider, S.J., Tischfield, D.J., Galazo, M.J. \& Macklis, J.D. RORbeta 963 induces barrel-like neuronal clusters in the developing neocortex. Cereb Cortex 22, 996-1006 964 (2012).

965 38. Oishi, K., Aramaki, M. \& Nakajima, K. Mutually repressive interaction between Brn1/2 966 and Rorb contributes to the establishment of neocortical layer 2/3 and layer 4. Proceedings of the 967 National Academy of Sciences of the United States of America 113, 3371-3376 (2016).

968 39. Nakagawa, Y. \& O'Leary, D.D. Dynamic patterned expression of orphan nuclear receptor 969 genes RORalpha and RORbeta in developing mouse forebrain. Dev Neurosci 25, 234-244 970 (2003).

971 40. Marinaro, F., et al. Molecular and cellular pathology of monogenic Alzheimer's disease 972 at single cell resolution. bioRxiv, 2020.2007.2014.202317 (2020).

973 41. Franjic, D., et al. Molecular Diversity Among Adult Human Hippocampal and Entorhinal 974 Cells. bioRxiv doi: https://doi.org/10.1101/2019.12.31.889139 (2019).

975 42. Heinsen, H., et al. Quantitative investigations on the human entorhinal area: left-right 976 asymmetry and age-related changes. Anat Embryol (Berl) 190, 181-194 (1994). 
977 43. Ehrenberg, A.J., et al. A manual multiplex immunofluorescence method for investigating 978 neurodegenerative diseases. Journal of Neuroscience Methods In press,

979 https://doi.org/10.1016/j.jneumeth.2020.108708. (2020).

980 44. Keren-Shaul, H., et al. A Unique Microglia Type Associated with Restricting

981 Development of Alzheimer's Disease. Cell 169, 1276-1290 e1217 (2017).

982 45. Srinivasan, K., et al. Alzheimer's patient brain myeloid cells exhibit enhanced aging and 983 unique transcriptional activation. bioRxiv doi: https://doi.org/10.1101/610345 (2019).

984 46. Thrupp, N., et al. Single nucleus sequencing fails to detect microglial activation in human 985 tissue. bioRxiv, 2020.2004.2013.035386 (2020).

986 47. Chen, W.T., et al. Spatial Transcriptomics and In Situ Sequencing to Study Alzheimer's 987 Disease. Cell (2020).

988 48. Chun, H. \& Lee, C.J. Reactive astrocytes in Alzheimer's disease: A double-edged sword. 989 Neurosci Res 126, 44-52 (2018).

990 49. Perez-Nievas, B.G. \& Serrano-Pozo, A. Deciphering the Astrocyte Reaction in 991 Alzheimer's Disease. Front Aging Neurosci 10, 114 (2018).

992 50. Simpson, J.E., et al. Microarray analysis of the astrocyte transcriptome in the aging brain: 993 relationship to Alzheimer's pathology and APOE genotype. Neurobiol Aging 32, 1795-1807

994 (2011).

995 51. Sekar, S., et al. Alzheimer's disease is associated with altered expression of genes 996 involved in immune response and mitochondrial processes in astrocytes. Neurobiol Aging 36, 997 583-591 (2015).

998 52. Liddelow, S.A., et al. Neurotoxic reactive astrocytes are induced by activated microglia. 999 Nature 541, 481-487 (2017).

1000 53. Brodkey, J.A., et al. Focal brain injury and upregulation of a developmentally regulated 1001 extracellular matrix protein. J Neurosurg 82, 106-112 (1995).

1002 54. Laywell, E.D., et al. Enhanced expression of the developmentally regulated extracellular 1003 matrix molecule tenascin following adult brain injury. Proceedings of the National Academy of 1004 Sciences of the United States of America 89, 2634-2638 (1992).

1005 55. Zamanian, J.L., et al. Genomic analysis of reactive astrogliosis. J Neurosci 32, 6391$10066410(2012)$.

1007 56. Anderson, M.A., et al. Astrocyte scar formation aids central nervous system axon 1008 regeneration. Nature 532, 195-200 (2016). 
1009 57. Insausti, R., Munoz-Lopez, M., Insausti, A.M. \& Artacho-Perula, E. The Human 1010 Periallocortex: Layer Pattern in Presubiculum, Parasubiculum and Entorhinal Cortex. A Review. 1011 Front Neuroanat 11, 84 (2017).

1012 58. Mikkonen, M., Alafuzoff, I., Tapiola, T., Soininen, H. \& Miettinen, R. Subfield- and 1013 layer-specific changes in parvalbumin, calretinin and calbindin-D28K immunoreactivity in the 1014 entorhinal cortex in Alzheimer's disease. Neuroscience 92, 515-532 (1999).

1015 59. Winterer, J., et al. Excitatory Microcircuits within Superficial Layers of the Medial 1016 Entorhinal Cortex. Cell Rep 19, 1110-1116 (2017).

1017 60. Braak, H. \& Braak, E. On areas of transition between entorhinal allocortex and temporal 1018 isocortex in the human brain. Normal morphology and lamina-specific pathology in Alzheimer's 1019 disease. Acta Neuropathol 68, 325-332 (1985).

1020 61. Kampmann, M. A CRISPR Approach to Neurodegenerative Diseases. Trends in 1021 molecular medicine 23, 483-485 (2017).

1022 62. Kampmann, M. CRISPR-based functional genomics for neurological disease. Nat Rev 1023 Neurol (2020).

1024 63. Tian, R., et al. CRISPR Interference-Based Platform for Multimodal Genetic Screens in 1025 Human iPSC-Derived Neurons. Neuron 104, 239-255 e212 (2019).

1026 64. Tian, R., et al. CRISPR Interference-Based Platform for Multimodal Genetic Screens in 1027 Human iPSC-Derived Neurons. Neuron 104, 239-255 (2019).

1028 65. Grinberg, L.T., et al. Brain bank of the Brazilian aging brain study group - a milestone 1029 reached and more than 1,600 collected brains. Cell Tissue Bank 8, 151-162 (2007).

1030 66. Hyman, B.T., et al. National Institute on Aging-Alzheimer's Association guidelines for 1031 the neuropathologic assessment of Alzheimer's disease. Alzheimers Dement 8, 1-13 (2012).

1032 67. Suemoto, C.K., et al. Neuropathological diagnoses and clinical correlates in older adults 1033 in Brazil: A cross-sectional study. PLoS Med 14, e1002267 (2017).

1034 68. Cairns, N.J., et al. Neuropathologic diagnostic and nosologic criteria for frontotemporal 1035 lobar degeneration: consensus of the Consortium for Frontotemporal Lobar Degeneration. Acta 1036 Neuropathol 114, 5-22 (2007).

1037 69. Ferrer, I., Santpere, G. \& van Leeuwen, F.W. Argyrophilic grain disease. Brain 131, 1038 1416-1432 (2008).

1039 70. Rodriguez, R.D. \& Grinberg, L.T. Argyrophilic grain disease: An underestimated 1040 tauopathy. Dement Neuropsychol 9, 2-8 (2015). 
1041 71. Rodriguez, R.D., et al. Argyrophilic Grain Disease: Demographics, Clinical, and 1042 Neuropathological Features From a Large Autopsy Study. J Neuropathol Exp Neurol 75, 6281043635 (2016).

1044 72. Mo, A., et al. Epigenomic Signatures of Neuronal Diversity in the Mammalian Brain. 1045 Neuron 86, 1369-1384 (2015).

1046 73. Tabula Muris, C., et al. Single-cell transcriptomics of 20 mouse organs creates a Tabula 1047 Muris. Nature 562, 367-372 (2018).

1048 74. Lun, A.T.L., et al. EmptyDrops: distinguishing cells from empty droplets in droplet1049 based single-cell RNA sequencing data. Genome biology 20, 63 (2019).

1050 75. Lun, A.T., Bach, K. \& Marioni, J.C. Pooling across cells to normalize single-cell RNA 1051 sequencing data with many zero counts. Genome biology 17, 75 (2016).

1052 76. Lun, A.T., McCarthy, D.J. \& Marioni, J.C. A step-by-step workflow for low-level 1053 analysis of single-cell RNA-seq data with Bioconductor. F1000Res 5, 2122 (2016).

1054 77. McCarthy, D.J., Campbell, K.R., Lun, A.T. \& Wills, Q.F. Scater: pre-processing, quality 1055 control, normalization and visualization of single-cell RNA-seq data in R. Bioinformatics 33, 1056 1179-1186 (2017).

1057 78. Stuart, T., et al. Comprehensive Integration of Single-Cell Data. Cell 177, 1888-1902 1058 e1821 (2019).

1059 79. Robinson, M.D., McCarthy, D.J. \& Smyth, G.K. edgeR: a Bioconductor package for 1060 differential expression analysis of digital gene expression data. Bioinformatics 26, 139-140 1061 (2010).

1062 80. Amezquita, R.A., et al. Orchestrating single-cell analysis with Bioconductor. Nat 1063 Methods 17, 137-145 (2020).

1064 81. Szklarczyk, D., et al. STRING v11: protein-protein association networks with increased 1065 coverage, supporting functional discovery in genome-wide experimental datasets. Nucleic acids 1066 research 47, D607-D613 (2019).

1067 82. Shannon, P., et al. Cytoscape: a software environment for integrated models of 1068 biomolecular interaction networks. Genome Res 13, 2498-2504 (2003).

1069 83. Cribari-Neto, F. \& Zeileis, A. Beta Regression in R. Journal of Statistical Software 34, 1107024 (2010).

1071 84. Stelzer, G., et al. The GeneCards Suite: From Gene Data Mining to Disease Genome 1072 Sequence Analyses. Curr Protoc Bioinformatics 54, 130 31-31 3033 (2016).

1073 85. Arriza, J.L., et al. Functional comparisons of three glutamate transporter subtypes cloned 1074 from human motor cortex. J Neurosci 14, 5559-5569 (1994). 
1075

1076

1077

1078

1079

1080

1081

1082

1083

1084

1085

1086

1087

1088

1089

1090

1091

1092

1093

1094

1095

1096

1097

1098

1099

1100

1101

1102

1103

1104

1105

1106

1107

86. Borden, L.A., et al. Cloning of the human homologue of the GABA transporter GAT-3 and identification of a novel inhibitor with selectivity for this site. Receptors Channels 2, 207213 (1994).

87. Gendreau, S., et al. A trimeric quaternary structure is conserved in bacterial and human glutamate transporters. J Biol Chem 279, 39505-39512 (2004).

88. Häberle, J., et al. Congenital glutamine deficiency with glutamine synthetase mutations. N Engl J Med 353, 1926-1933 (2005).

89. Kawakami, H., Tanaka, K., Nakayama, T., Inoue, K. \& Nakamura, S. Cloning and expression of a human glutamate transporter. Biochem Biophys Res Commun 199, 171-176 (1994).

90. Melzer, N., Biela, A. \& Fahlke, C. Glutamate modifies ion conduction and voltagedependent gating of excitatory amino acid transporter-associated anion channels. J Biol Chem 278, 50112-50119 (2003).

91. Südhof, T.C. Synaptic Neurexin Complexes: A Molecular Code for the Logic of Neural Circuits. Cell 171, 745-769 (2017).

92. Pellissier, F., Gerber, A., Bauer, C., Ballivet, M. \& Ossipow, V. The adhesion molecule Necl-3/SynCAM-2 localizes to myelinated axons, binds to oligodendrocytes and promotes cell adhesion. BMC Neurosci 8, 90 (2007).

93. González-Castillo, C., Ortuño-Sahagún, D., Guzmán-Brambila, C., Pallàs, M. \& RojasMayorquín, A.E. Pleiotrophin as a central nervous system neuromodulator, evidences from the hippocampus. Front Cell Neurosci 8, 443 (2014).

94. Siddiqui, T.J., et al. An LRRTM4-HSPG complex mediates excitatory synapse development on dentate gyrus granule cells. Neuron 79, 680-695 (2013).

95. Heinsen, H., Arzberger, T. \& Schmitz, C. Celloidin mounting (embedding without infiltration) - a new, simple and reliable method for producing serial sections of high thickness through complete human brains and its application to stereological and immunohistochemical investigations. J Chem Neuroanat 20, 49-59 (2000).

96. Insausti, R. \& Amaral, D.G. Entorhinal cortex of the monkey: IV. Topographical and laminar organization of cortical afferents. J Comp Neurol 509, 608-641 (2008).

97. Rose, S. Vergleichende Messungen im Allocortex bei Tier und Mensch. J Psychol Neurol 34, 250-255 (1927).

98. Hevner, R.F., et al. Tbr1 regulates differentiation of the preplate and layer 6. Neuron 29, 353-366 (2001). 
1108 99. Ehrenberg, A.J., et al. Quantifying the accretion of hyperphosphorylated tau in the locus 1109 coeruleus and dorsal raphe nucleus: the pathological building blocks of early Alzheimer's 1110 disease. Neuropathology and applied neurobiology 43, 393-408 (2017).

1111 100. Montine, T.J., et al. National Institute on Aging-Alzheimer's Association guidelines for 1112 the neuropathologic assessment of Alzheimer's disease: a practical approach. Acta Neuropathol 1113 123, 1-11 (2012).

1114 101. Hughes, C.P., Berg, L., Danziger, W.L., Coben, L.A. \& Martin, R.L. A new clinical scale 1115 for the staging of dementia. Br J Psychiatry 140, 566-572 (1982).

1116

1117 


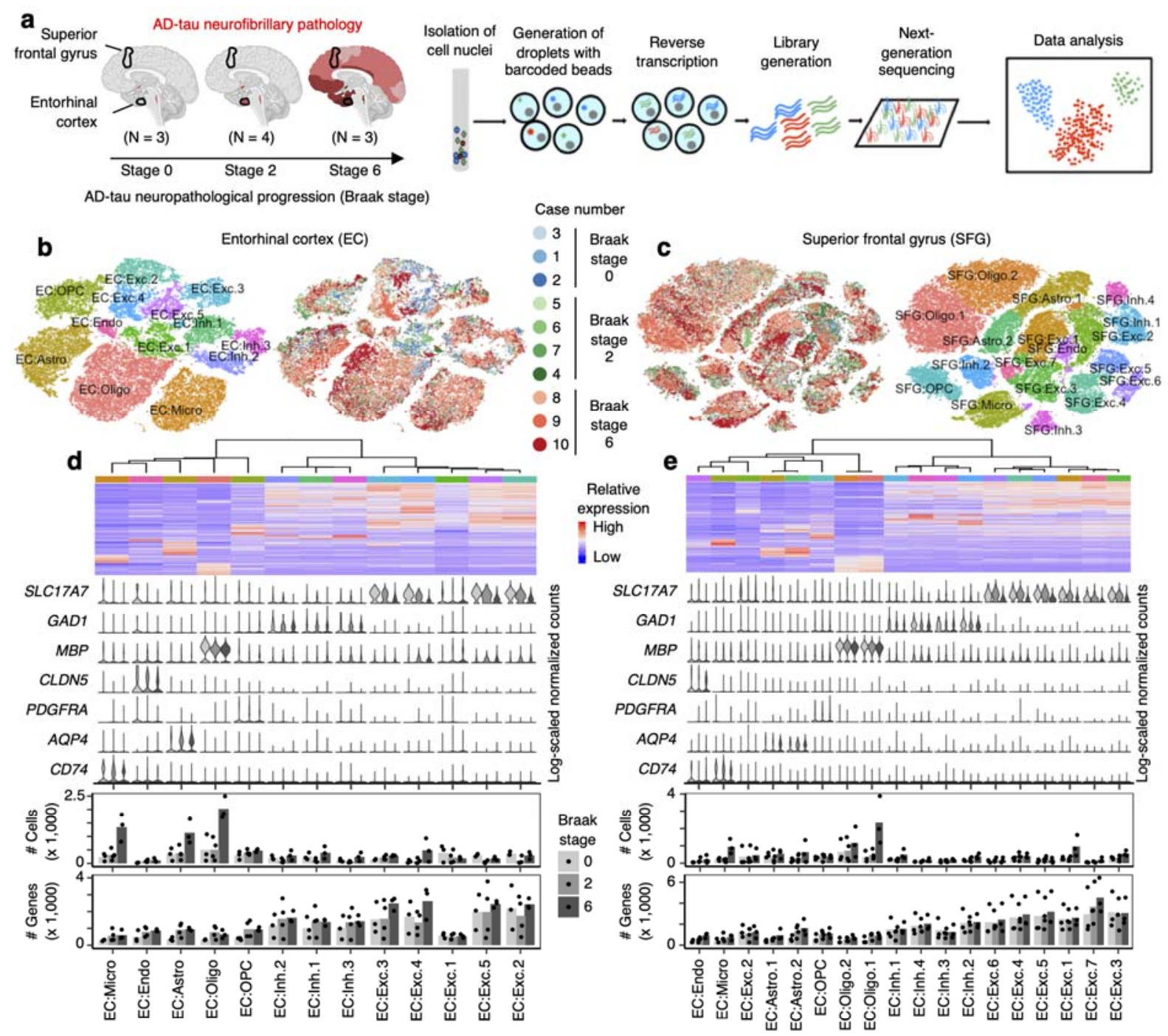

f

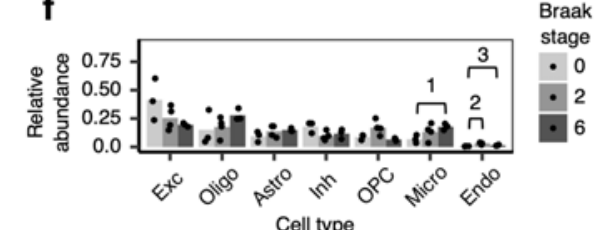

\begin{tabular}{|cc|}
\hline Comparison & $P_{\text {adjusted }}$ \\
\hline 1 & 0.08 \\
\hline 2 & $5 \times 10^{-10}$ \\
\hline 3 & 0.03 \\
\hline
\end{tabular}

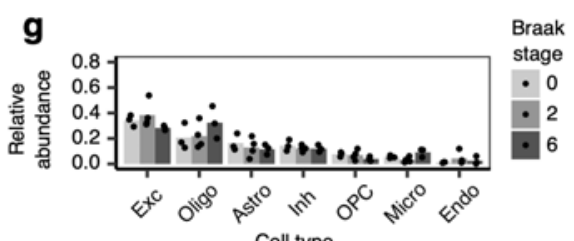

Fig. 1 AD progression differentially affects the cell-type composition of the EC and SFG. a, Schematic of experimental design and sample processing. Darker shades of red in brain cartoons reflect more severe $\mathrm{AD}$-tau neurofibrillary pathology. b-c, tSNE projection of cells from the EC (b) and SFG (c) in their respective alignment spaces, colored by individual of origin (center) or cluster assignment (outer). d-e, Heatmap and hierarchical clustering of clusters and cluster marker expression (top subpanel); "High" and "Low" relative expression reflect above- and below-average expression, respectively (see Methods). Expression of cell type markers in each cluster (second subpanel). The average number of cells and average number of genes detected per cell in each cluster (third and fourth subpanels). f-g, Relative abundance of major cell types across Braak stages. Cell type abbreviations: Exc - excitatory neurons, Oligo oligodendrocytes, Astro - astrocytes, Inh - inhibitory neurons, OPC - oligodendrocyte precursor cells, Micro - microglia, Endo - endothelial cells. 
bioRxiv preprint doi: https://doi.org/10.1101/2020.04.04.025825; this version posted August 23, 2020. The copyright holder for this preprint (which was not certified by peer review) is the author/funder. All rights reserved. No reuse allowed without permission.

1131 

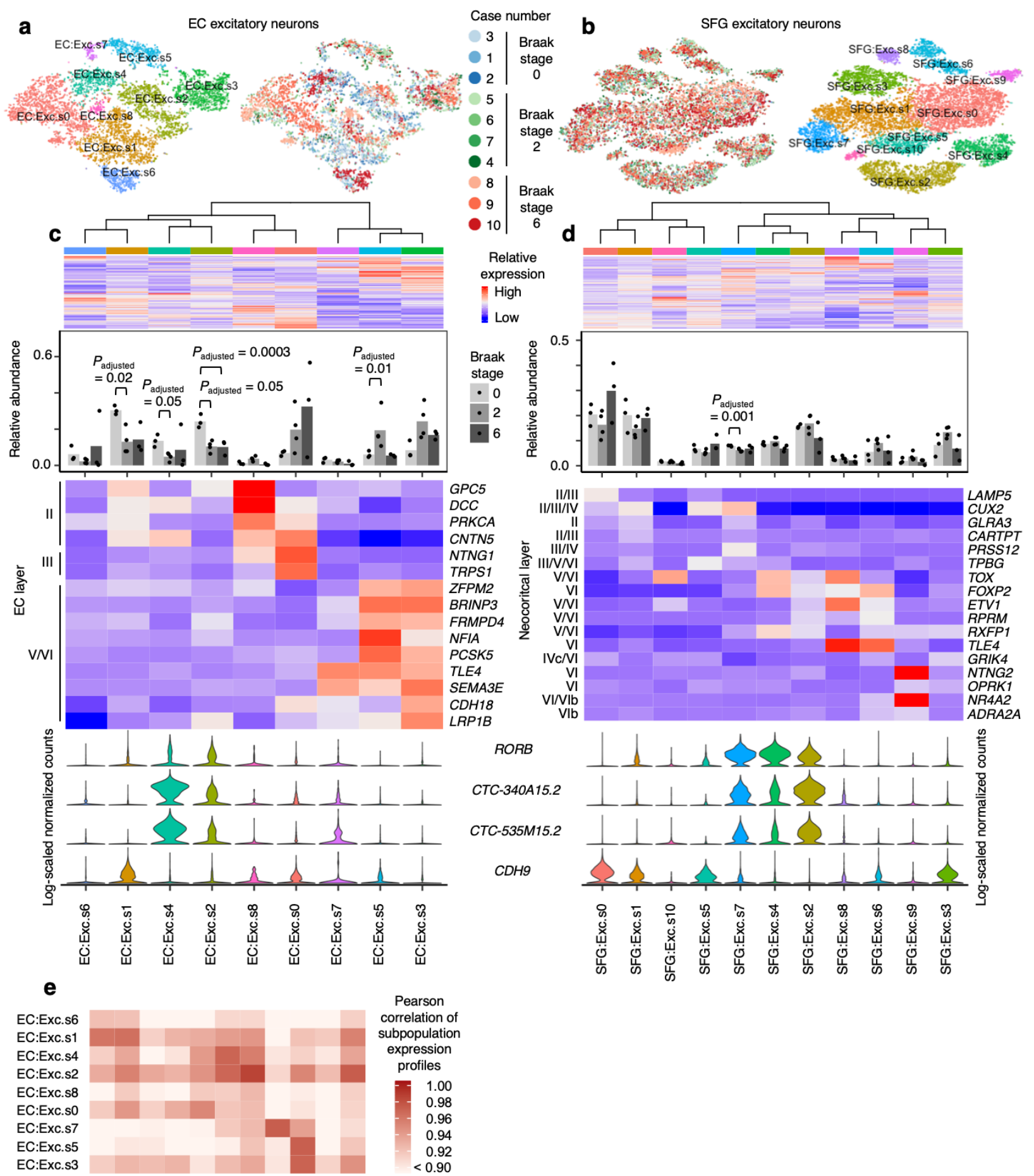
vulnerable. a-b, tSNE projection of excitatory neurons from the EC (a) and SFG (b) in their respective alignment spaces, colored by individual of origin (center) or subpopulation identity 1136 (outer). c-d, Heatmap and hierarchical clustering of subpopulations and subpopulation marker 1137 expression (top subpanel); "High" and "Low" relative expression reflect above- and below- 
1138 average expression, respectively (see Methods). Relative abundance of subpopulations across 1139 Braak stages (second subpanel). Expression heatmap of EC layer-specific genes identified from 1140 Ramsden et al. ${ }^{34}$ (c, third subpanel). Expression heatmap of neocortical layer-specific genes from 1141 Lake et al. ${ }^{19}$ (d, third subpanel). Expression of selectively vulnerable subpopulation markers 1142 identified in the EC (bottom subpanel). e, Heatmap of Pearson correlation between the gene 1143 expression profiles of EC and SFG subpopulations. 

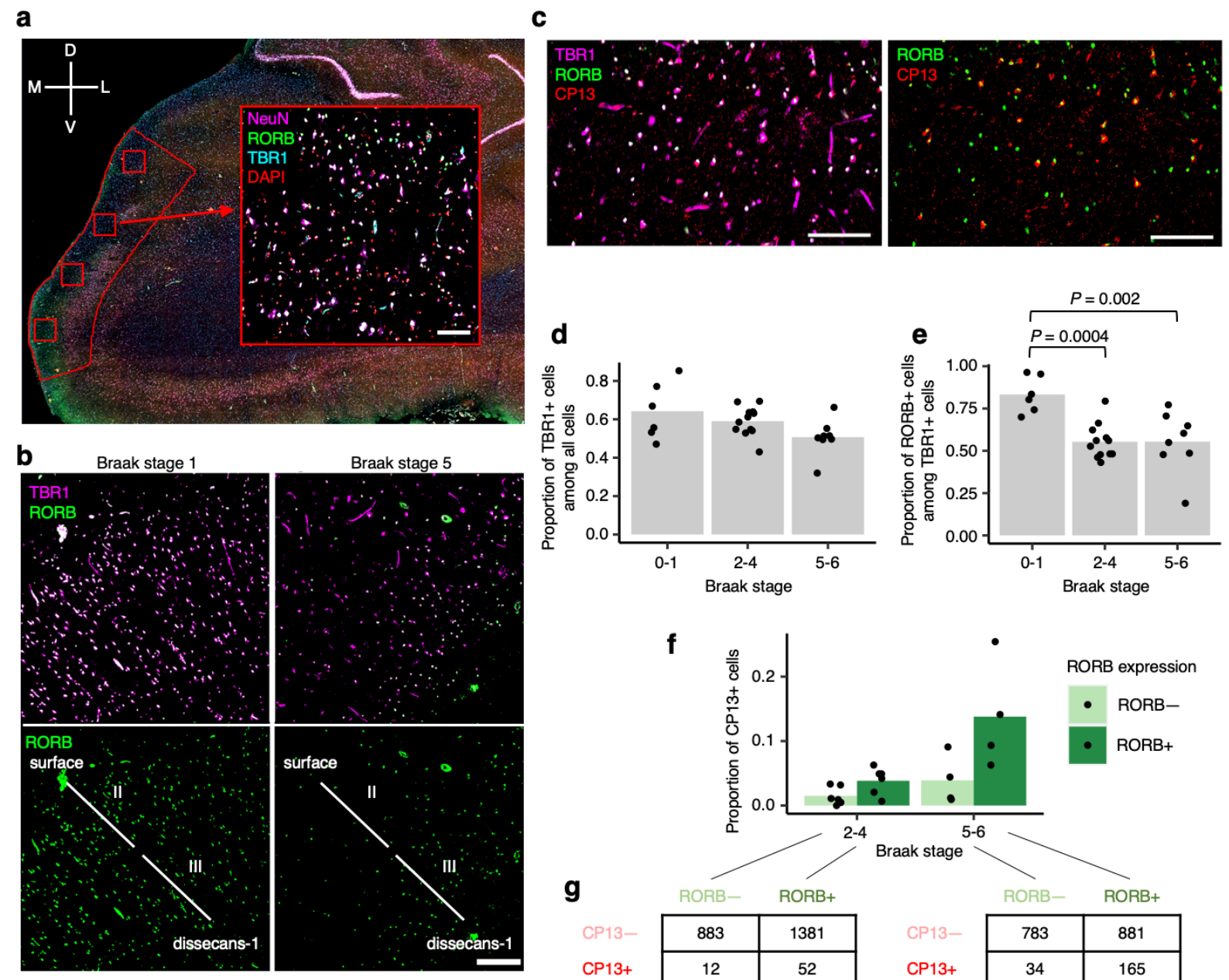

h
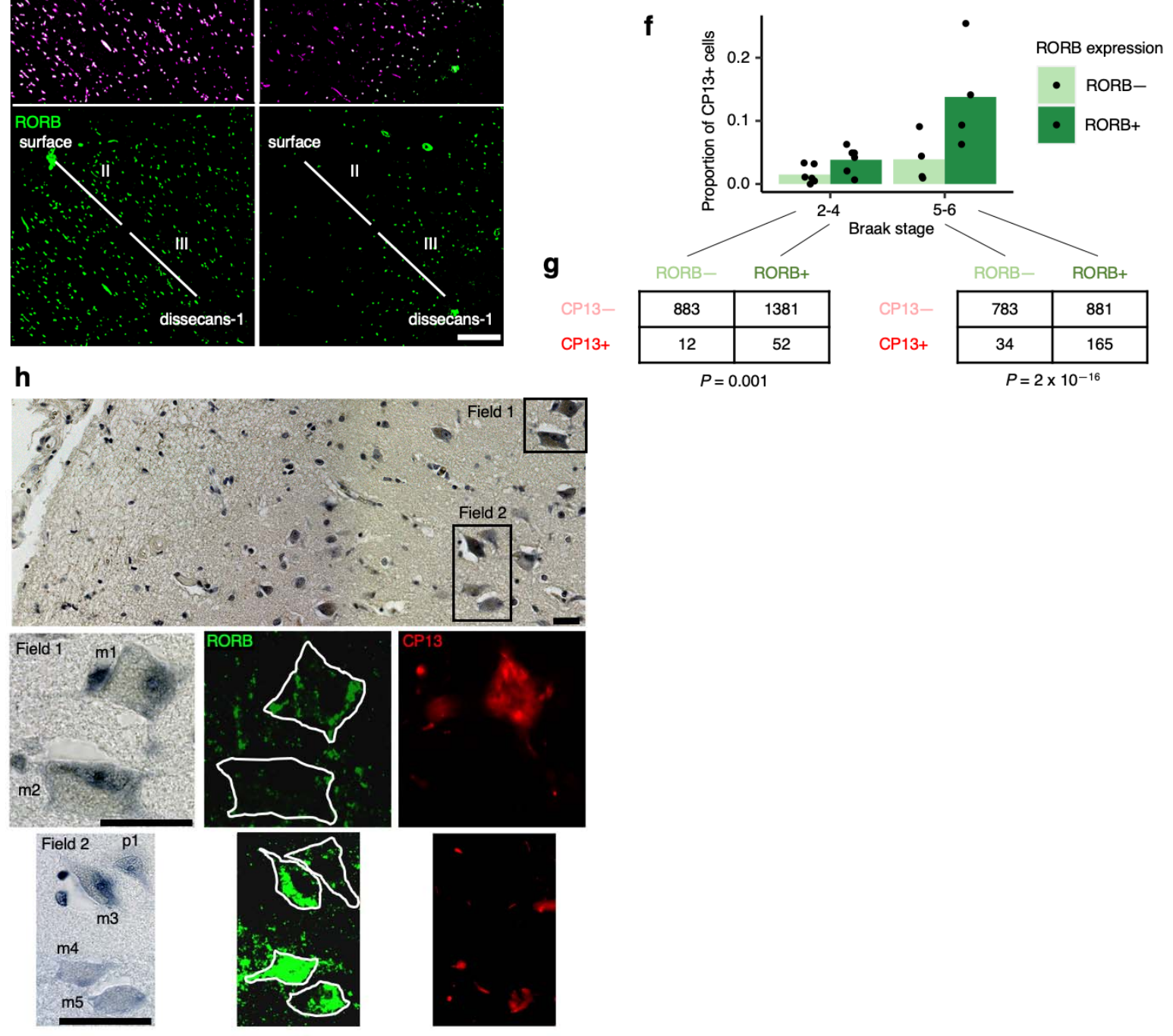
1146 Fig. 3 | Immunofluorescence of the EC validates selective vulnerability of RORB-expressing 1147 excitatory neurons. a, The method for extracting regions of interest (ROI) is illustrated using a 1148 representative brain slice used for immunofluorescence with the EC delineated in red. Four ROIs 1149 (drawn in red squares) were randomly distributed along the superficial layers of the EC and 1150 extracted for quantification after masking neurons (see Methods). A representative ROI image 1151 with DAPI, NeuN, TBR1, and RORB staining is shown. The anatomical orientation of the slice 1152 is provided in the top left corner ( $\mathrm{D}$ - dorsal, V - ventral, $\mathrm{M}$ - medial, $\mathrm{L}$ - lateral). b,

1153 Representative RORB staining in a Braak stage 1 sample (left) vs. a Braak stage 5 sample (right), 1154 shown with (top) and without (bottom) excitatory neurons marked by TBR1 staining. The EC 1155 layers captured in the image are demarcated in the bottom subpanels (see Methods and Extended 1156 Data Fig. 6). c, Representative CP13 staining in a Braak stage 6 sample, shown together with 1157 TBR1 and RORB staining (left) or only with RORB staining (right). d-e, Proportion of TBR1+ 1158 cells among all cells $(\mathbf{d})$ or proportion of RORB + cells among TBR1+ cells (e) averaged across 1159 ROIs for each individual across groups of Braak stages. f, Proportion of CP13+ cells in RORB1160 or RORB+ excitatory neurons (i.e. TBR1+ cells) averaged across ROIs for each individual 1161 across groups of Braak stages. $\mathrm{g}$, Contingency tables of raw counts of TBR1+ cells based on 1162 their RORB or CP13 staining status summed across ROIs and individuals for each group of 1163 Braak stages; the Fisher's Exact Test p-value is shown below each table. h, Representative image 1164 of EC layer II neurons stained with gallocyanin (top subpanel) with the corresponding RORB 1165 and CP13 immunofluorescence signal shown in selected fields (Field 1 - middle subpanels, Field 11662 - bottom subpanels). RORB+ neurons include both large multipolar neurons (m1, m3, m4, m5) 1167 and pyramidal neurons (p1). One large multipolar neuron (m2) is RORB-. The neuronal somas 1168 are outlined manually in white in the RORB immunofluorescence images to aid interpretation. 1169 Scale bars shown in a-c correspond to 100 microns; scale bars shown in $\mathbf{h}$ correspond to 15 1170 microns. 
1173
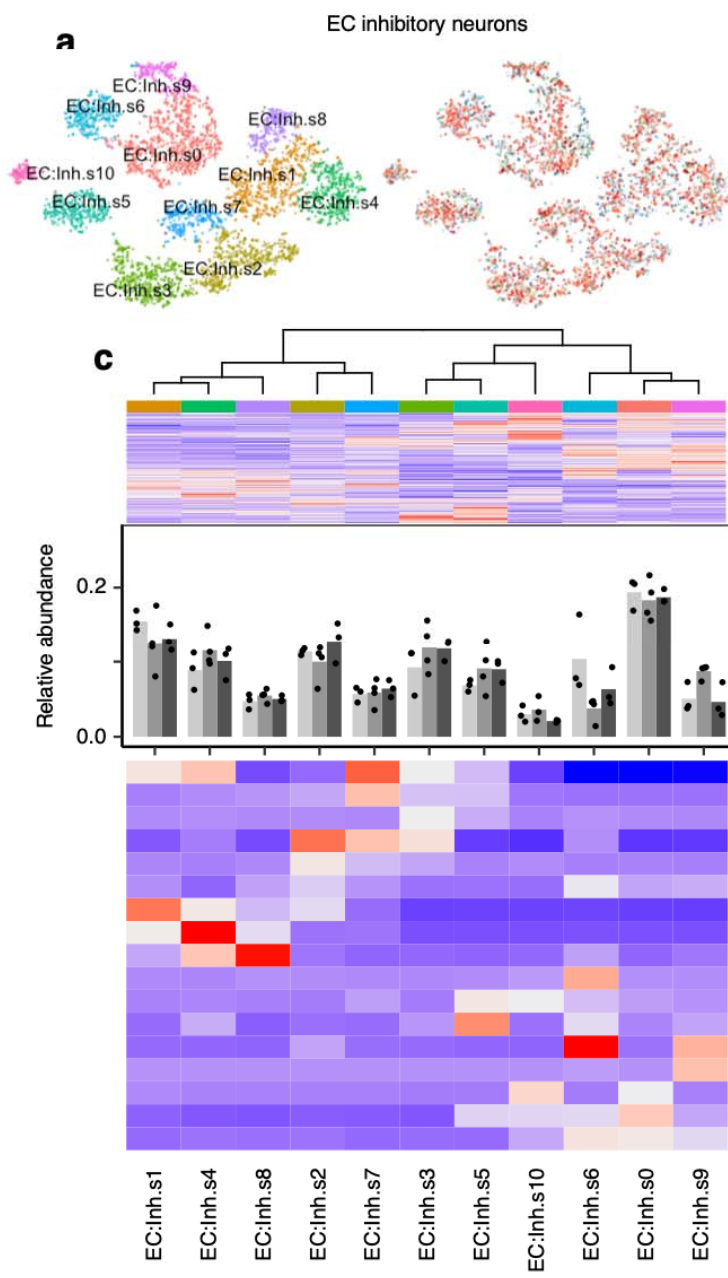
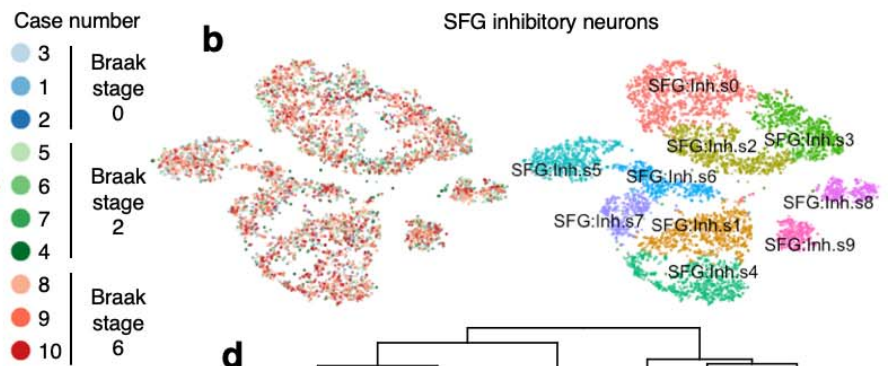

106

\section{Relative expression High \\ Low}

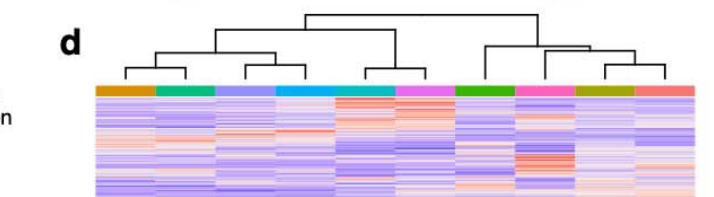

Braak stage

- 0

- 2

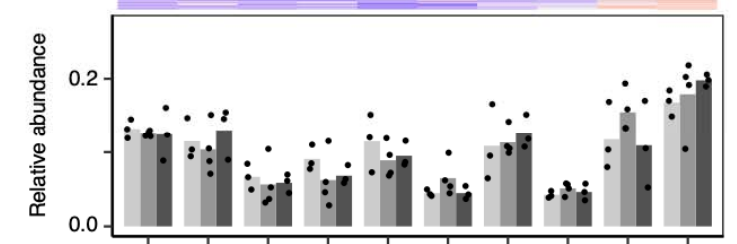

CCK

TNFAIP8L3 TNFAIP8L3
PDYN

PDYN

RELN

NDNF

CALB2

VIP

TAC3
NPY

CALB1

NOS1

SST

TH

TAC1

TAC1
CRHBP

$C C K$
TNFAIP8L3
PDYN
RELN
NDNF
PNOC
CALB2
VIP
TAC3
NPY
CALB1
NOS1
SST
TH
PVALB
TAC1
CRHBP

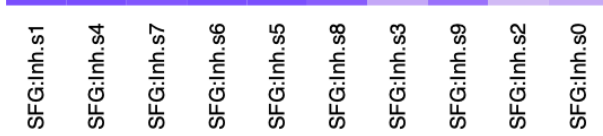

Fig. 4 | Inhibitory neuron subpopulations do not consistently show differences in resilience or vulnerability to AD progression. a-b, tSNE projection of inhibitory neurons from the EC (a) and SFG (b) in their respective alignment spaces, colored by individual of origin (center) or subpopulation identity (outer). c-d, Heatmap and hierarchical clustering of subpopulations and subpopulation marker expression (top subpanel); "High" and "Low" relative expression reflect above- and below-average expression, respectively (see Methods). Relative abundance of subpopulations across Braak stages (middle subpanel). Expression heatmap of inhibitory neuron molecular subtype markers from Lake et al. ${ }^{19}$ (bottom subpanel). 


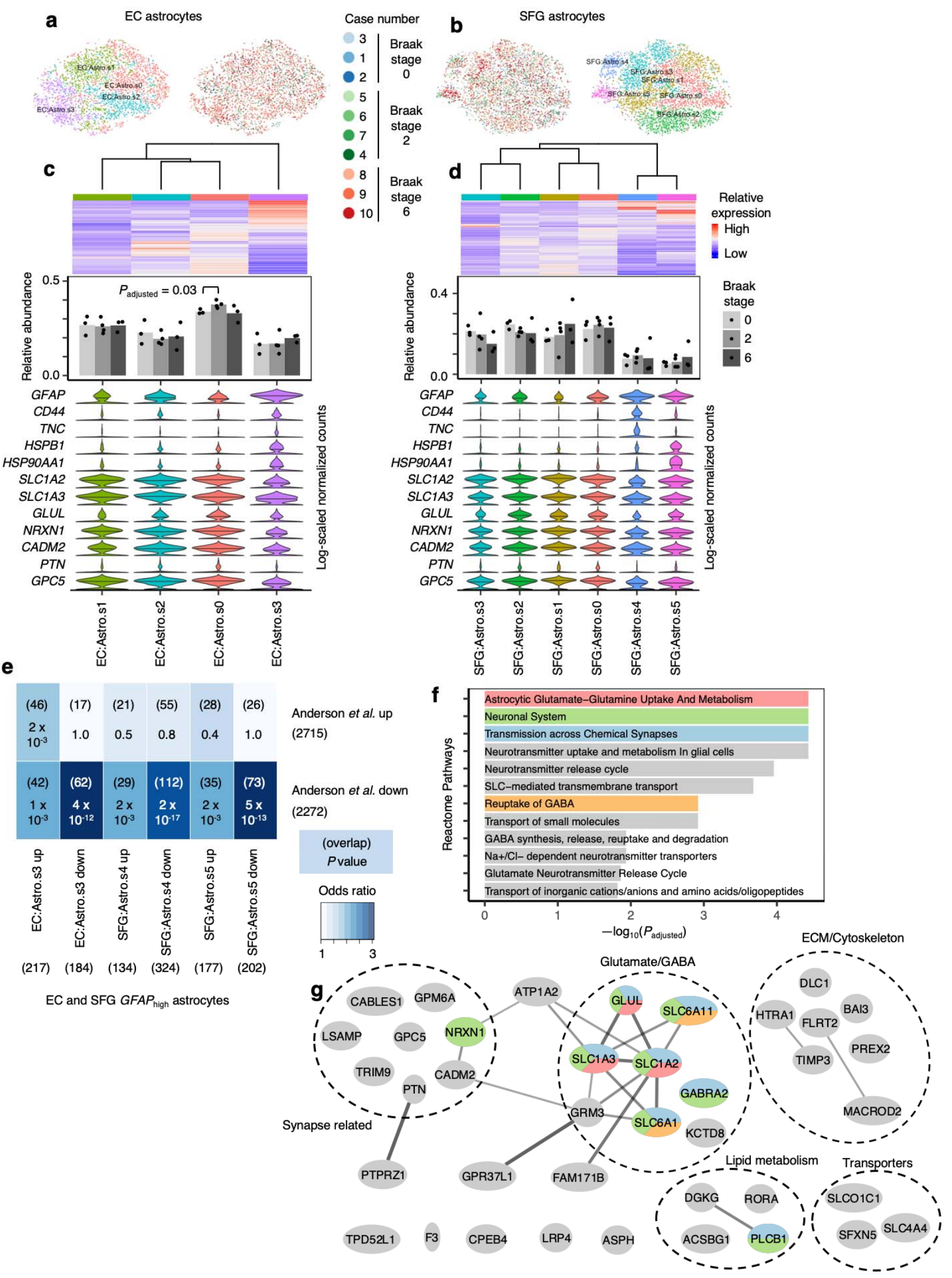


1186 synaptic support. a-b, tSNE projection of astrocytes from the EC (a) and SFG (b) in their 1187 respective alignment spaces, colored by individual of origin (center) or subpopulation identity 1188 (outer). c-d, Heatmap and hierarchical clustering of subpopulations and subpopulation marker 1189 expression (top subpanel); "High" and "Low" relative expression reflect above- and below1190 average expression, respectively (see Methods). Relative abundance of subpopulations across 1191 Braak stages (middle subpanel). Expression of genes associated with reactive astrocytes, with 1192 median expression level marked by line (bottom subpanel). e, Enrichment analysis of overlap 1193 between differentially expressed genes in $G F A P_{\text {high }}$ astrocytes vs. differentially expressed genes 1194 in reactive astrocytes from Anderson et al. ${ }^{56}$ The number of genes in each gene set and the 1195 number of overlapping genes are shown in parentheses, and the hypergeometric test p-values 1196 (corrected for multiple testing using the Benjamini-Hochberg procedure) are shown without 1197 parentheses. f, Enrichment of Reactome pathways in downregulated genes in $G F A P_{\text {high }}$ 1198 astrocytes, with selected terms highlighted in color. g, Functional association network (see 1199 Methods) of downregulated genes shared between EC and SFG GFAP ${ }_{\text {high }}$ astrocytes that overlap 1200 with those in Anderson et al. ${ }^{56}$ Genes with stronger associations are connected by thicker lines. 1201 Genes that belong to selected gene sets in $\mathbf{k}$ are highlighted in color. 
TABLE 1

Cases used for snRNA-seq

\begin{tabular}{|c|c|c|c|c|c|c|c|c|}
\hline $\begin{array}{c}\text { Case } \\
\#\end{array}$ & $\begin{array}{c}\text { Braak } \\
\text { stage }\end{array}$ & Sex & $\begin{array}{l}\text { Age at } \\
\text { death } \\
\text { (years) }\end{array}$ & $\begin{array}{c}\text { Post- } \\
\text { mortem } \\
\text { interval } \\
\text { (hours) }\end{array}$ & $\begin{array}{c}\text { ADNC } \\
\text { score }\end{array}$ & $\begin{array}{c}\text { CDR } \\
\text { before } \\
\text { death }\end{array}$ & $\begin{array}{c}A P O E \\
\text { genotype }\end{array}$ & Source \\
\hline 1 & 0 & $\mathrm{M}$ & 50 & 13 & $\mathrm{~A} 0, \mathrm{~B} 0, \mathrm{C} 0$ & 0 & E3/E3 & BBAS \\
\hline 2 & 0 & $\mathrm{M}$ & 60 & 12 & $\mathrm{~A} 0, \mathrm{~B} 0, \mathrm{C} 0$ & 0.5 & E3/E3 & BBAS \\
\hline 3 & 0 & $\mathrm{M}$ & 71 & 12 & $\mathrm{~A} 1, \mathrm{~B} 0, \mathrm{C} 0$ & 0 & E3/E3 & BBAS \\
\hline 4 & 2 & $\mathrm{M}$ & 72 & 15 & $\mathrm{~A} 1, \mathrm{~B} 1, \mathrm{C} 0$ & 0 & E3/E3 & BBAS \\
\hline $5^{*}$ & 2 & $\mathrm{M}$ & 77 & 4.9 & $\mathrm{~A} 2, \mathrm{~B} 1, \mathrm{C} 1$ & 0.5 & E3/E3 & UCSF \\
\hline $6^{*}$ & 2 & $\mathrm{M}$ & 87 & 30 & $\mathrm{~A} 2, \mathrm{~B} 1, \mathrm{C} 2$ & 2 & E3/E3 & UCSF \\
\hline $7 *$ & 2 & $\mathrm{M}$ & 91 & 50 & $\mathrm{~A} 1, \mathrm{~B} 1, \mathrm{C} 1$ & 0 & E3/E3 & UCSF \\
\hline $8^{*}$ & 6 & $\mathrm{M}$ & 72 & 6.9 & $\mathrm{~A} 3, \mathrm{~B} 3, \mathrm{C} 3$ & 3 & E3/E3 & UCSF \\
\hline $9 *$ & 6 & $\mathrm{M}$ & 82 & 6.7 & $\mathrm{~A} 3, \mathrm{~B} 3, \mathrm{C} 3$ & 3 & E3/E3 & UCSF \\
\hline 10 & 6 & $\mathrm{M}$ & 82 & 9 & $\mathrm{~A} 3, \mathrm{~B} 3, \mathrm{C} 3$ & 3 & E3/E3 & UCSF \\
\hline \multicolumn{9}{|c|}{ Cases used for immunofluorescence validation } \\
\hline $\begin{array}{c}\text { Case } \\
\#\end{array}$ & $\begin{array}{c}\text { Braak } \\
\text { stage }\end{array}$ & Sex & $\begin{array}{c}\text { Age at } \\
\text { death }\end{array}$ & $\begin{array}{c}\text { Post- } \\
\text { mortem } \\
\text { interval } \\
\text { (hours) }\end{array}$ & $\begin{array}{c}\text { ADNC } \\
\text { score }\end{array}$ & $\begin{array}{c}\text { CDR } \\
\text { before } \\
\text { death }\end{array}$ & $\begin{array}{c}A P O E \\
\text { genotype }\end{array}$ & Source \\
\hline $5^{*}$ & 2 & $\mathrm{M}$ & 77 & 4.9 & $\mathrm{~A} 2, \mathrm{~B} 1, \mathrm{C} 1$ & 0.5 & E3/E3 & UCSF \\
\hline $6 *$ & 2 & $\mathrm{M}$ & 87 & 30 & $\mathrm{~A} 2, \mathrm{~B} 1, \mathrm{C} 2$ & 2 & E3/E3 & UCSF \\
\hline $7 *$ & 2 & $\mathrm{M}$ & 91 & 50 & $\mathrm{~A} 1, \mathrm{~B} 1, \mathrm{C} 1$ & 0 & E3/E3 & UCSF \\
\hline $8^{*}$ & 6 & $\mathrm{M}$ & 72 & 6.9 & $\mathrm{~A} 3, \mathrm{~B} 3, \mathrm{C} 3$ & 3 & E3/E3 & UCSF \\
\hline $9 *$ & 6 & $\mathrm{M}$ & 82 & 6.7 & $\mathrm{~A} 3, \mathrm{~B} 3, \mathrm{C} 3$ & 3 & E3/E3 & UCSF \\
\hline 11 & 0 & $\mathrm{~F}$ & 62 & 10.1 & $\mathrm{~A} 1, \mathrm{~B} 0, \mathrm{C} 0$ & 0 & NA & BBAS \\
\hline 12 & 0 & $\mathrm{M}$ & 64 & 12 & $\mathrm{~A} 0, \mathrm{~B} 0, \mathrm{C} 0$ & 0 & E3/E3 & BBAS \\
\hline 13 & 1 & $\mathrm{M}$ & 60 & 19 & $\mathrm{~A} 0, \mathrm{~B} 1, \mathrm{C} 0$ & 0 & NA & BBAS \\
\hline 14 & 1 & $\mathrm{~F}$ & 64 & 13 & $\mathrm{~A} 1, \mathrm{~B} 1, \mathrm{C} 0$ & 0 & E3/E3 & BBAS \\
\hline 15 & 1 & $\mathrm{M}$ & 70 & 11 & $\mathrm{~A} 1, \mathrm{~B} 1, \mathrm{C} 0$ & 0 & E3/E3 & BBAS \\
\hline 16 & 1 & $\mathrm{~F}$ & 82 & 9.6 & $\mathrm{~A} 1, \mathrm{~B} 1, \mathrm{C} 0$ & 0 & NA & BBAS \\
\hline 17 & 2 & $\mathrm{~F}$ & 79 & 18 & $\mathrm{~A} 1, \mathrm{~B} 1, \mathrm{C} 1$ & 0 & E3/E3 & BBAS \\
\hline 18 & 2 & $\mathrm{~F}$ & 81 & 30.3 & $\mathrm{~A} 1, \mathrm{~B} 1, \mathrm{C} 0$ & NA & E3/E3 & UCSF \\
\hline 19 & 3 & $\mathrm{M}$ & 81 & 8.3 & $\mathrm{~A} 2, \mathrm{~B} 2, \mathrm{C} 3$ & 1 & NA & UCSF \\
\hline 20 & 3 & $\mathrm{M}$ & 84 & 28 & $\mathrm{~A} 3, \mathrm{~B} 2, \mathrm{C} 2$ & 1 & NA & UCSF \\
\hline 21 & 3 & $\mathrm{~F}$ & 88 & 9.8 & $\mathrm{~A} 3, \mathrm{~B} 2, \mathrm{C} 2$ & 0.5 & E3/E3 & UCSF \\
\hline 22 & 3 & $\mathrm{M}$ & 89 & 9.1 & $\mathrm{~A} 3, \mathrm{~B} 2, \mathrm{C} 2$ & 1 & E3/E3 & UCSF \\
\hline
\end{tabular}




\begin{tabular}{|c|c|c|c|c|c|c|c|c|}
\hline 23 & 4 & $\mathrm{~F}$ & 87 & 9.5 & $\mathrm{~A} 1, \mathrm{~B} 2, \mathrm{C} 3$ & 2 & E3/E3 & UCSF \\
\hline 24 & 4 & M & 91 & 11.2 & $\mathrm{~A} 3, \mathrm{~B} 2, \mathrm{C} 2$ & 0.5 & E3/E3 & UCSF \\
\hline 25 & 4 & M & 103 & 7.8 & $\mathrm{~A} 1, \mathrm{~B} 2, \mathrm{C} 2$ & NA & E3/E3 & UCSF \\
\hline 26 & 5 & $\mathrm{M}$ & 77 & 8.4 & $\mathrm{~A} 3, \mathrm{~B} 3, \mathrm{C} 3$ & 0.5 & E4/E4 & UCSF \\
\hline 27 & 5 & M & 85 & 11.2 & $\mathrm{~A} 3, \mathrm{~B} 3, \mathrm{C} 3$ & 1 & E3/E3 & UCSF \\
\hline 28 & 5 & M & 86 & 8.6 & $\mathrm{~A} 3, \mathrm{~B} 3, \mathrm{C} 3$ & 2 & E3/E4 & UCSF \\
\hline 29 & 5 & $\mathrm{~F}$ & 87 & 17 & $\mathrm{~A} 3, \mathrm{~B} 3, \mathrm{C} 2$ & 3 & E3/E3 & BBAS \\
\hline 30 & 6 & $\mathrm{~F}$ & 64 & 7.3 & $\mathrm{~A} 3, \mathrm{~B} 3, \mathrm{C} 3$ & 3 & E3/E4 & UCSF \\
\hline 31 & 6 & $\mathrm{~F}$ & 67 & 9.7 & $\mathrm{~A} 3, \mathrm{~B} 3, \mathrm{C} 3$ & 3 & E4/E4 & UCSF \\
\hline
\end{tabular}

\section{Table 1 | Description of post-mortem cohort.}

1209 Asterisks denote cases used both for snRNA-seq and immunofluorescence validation. The AD neuropathological change (ADNC) score incorporates assessment of amyloid-beta deposits

1211 ("A"), staging of neurofibrillary tangles ("B"), and scoring of neuritic plaques ("C" $C)^{100}$. The 1212 Clinical Dementia Rating (CDR) reflects the degree of cognitive impairment ${ }^{101}$. 
a
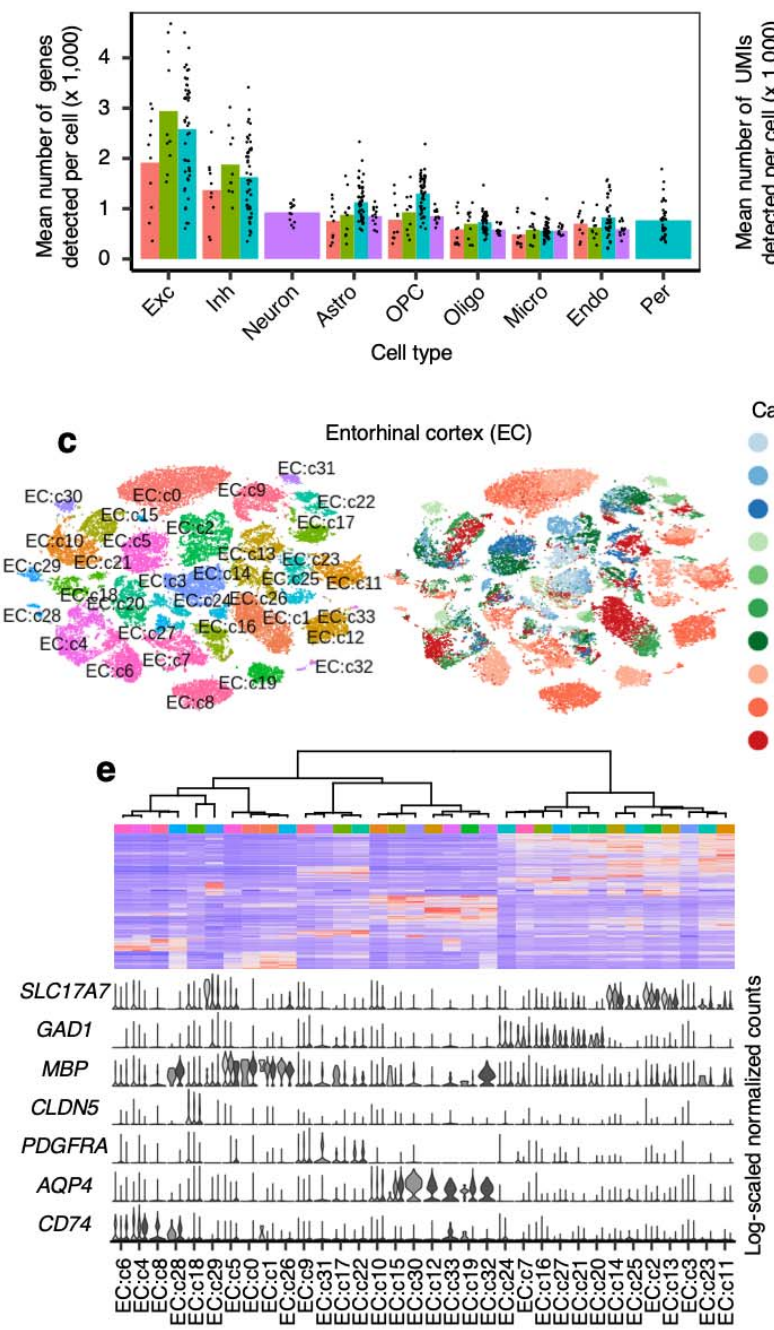

b
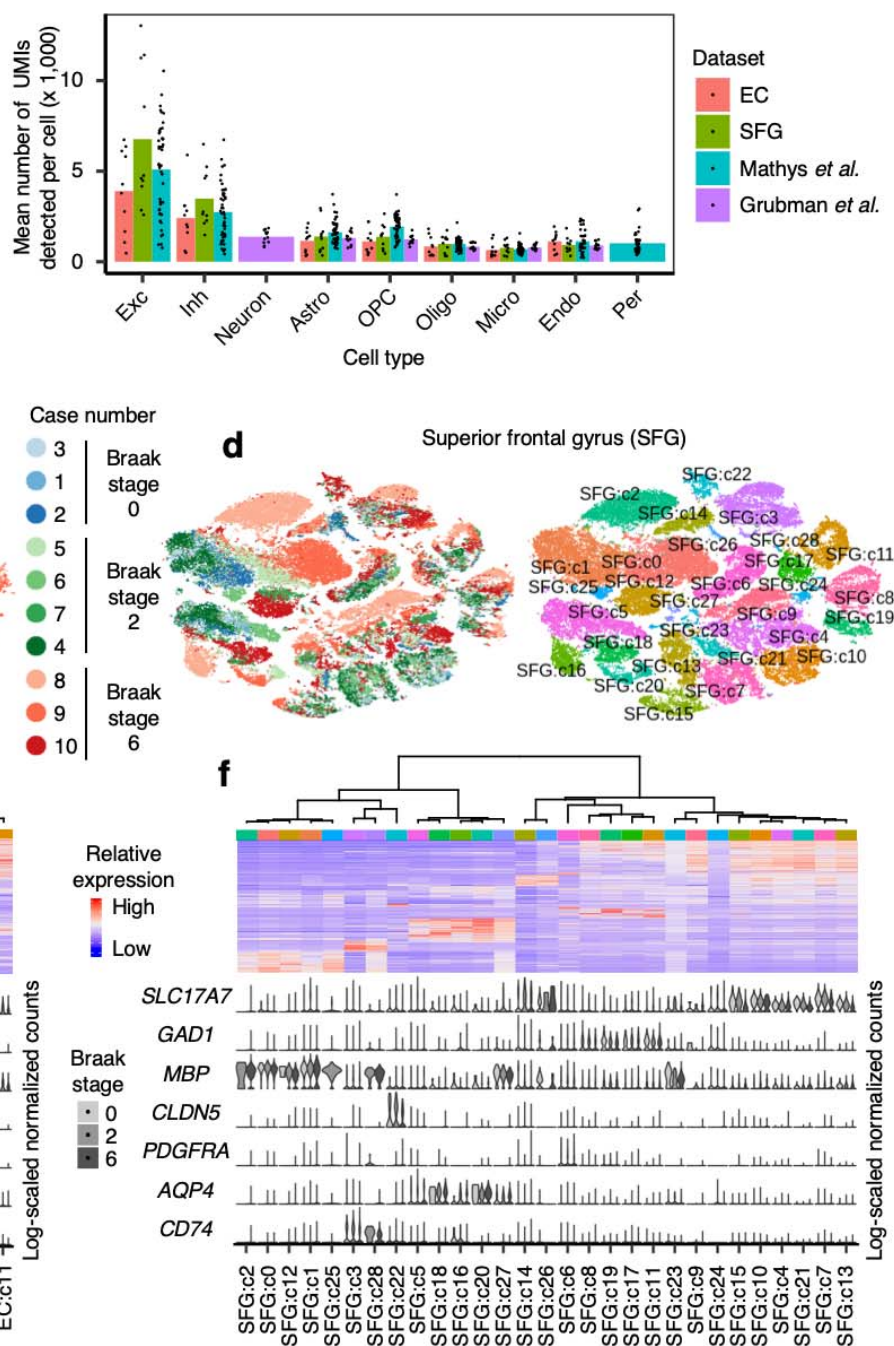

Extended Data Fig. 1 | Data quality and initial clustering without cross-sample alignment. $\mathbf{a}-\mathbf{b}$, Mean number of genes (a) or UMIs (b) detected per cell across individual samples for major cell types identified in each dataset. Grubman et al. ${ }^{22}$ did not resolve excitatory neurons from inhibitory neurons. Pericytes were identified only in Mathys et al. ${ }^{21}$ Cell type abbreviations: Exc - excitatory neurons, Oligo - oligodendrocytes, Astro - astrocytes, Inh - inhibitory neurons, OPC - oligodendrocyte precursor cells, Micro - microglia, Endo - endothelial cells, Per pericytes. c-d, tSNE projection of cells from the EC (c) and SFG (d) clustered without first performing cross-sample alignment, colored by individual of origin (center) or cluster assignment (outer). e-f, Heatmap and hierarchical clustering of clusters and cluster marker expression (top subpanels); "High" and "Low" relative expression reflect above- and belowaverage expression, respectively (see Methods). Expression of cell type markers (bottom subpanels). 
bioRxiv preprint doi: https://doi.org/10.1101/2020.04.04.025825; this version posted August 23, 2020. The copyright holder for this preprint (which was not certified by peer review) is the author/funder. All rights reserved. No reuse allowed without permission.

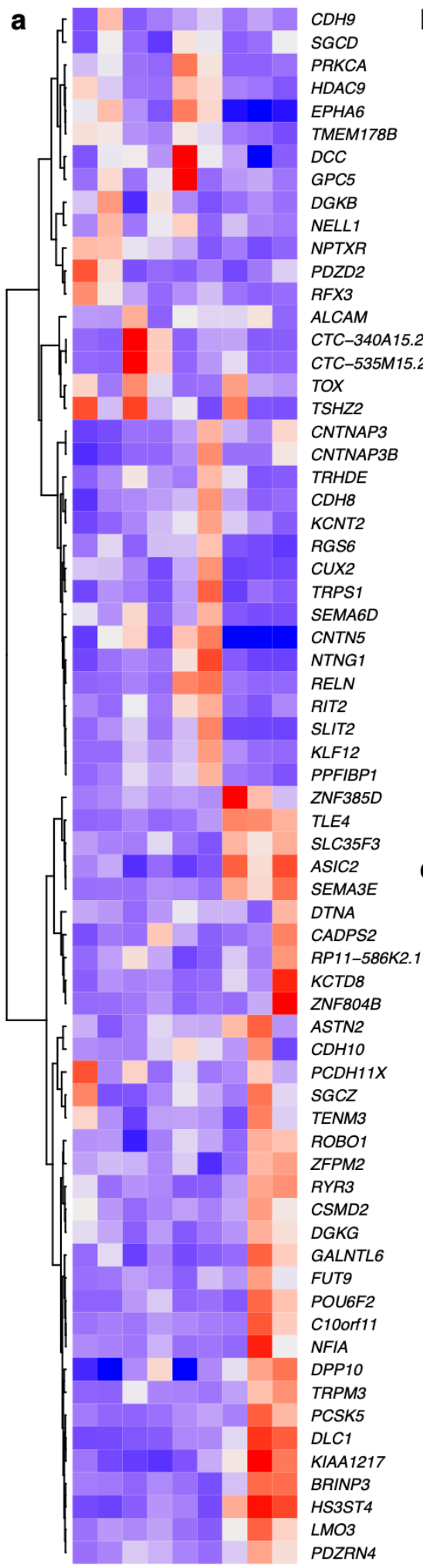

Relative

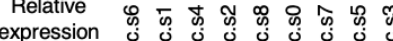

High $\quad$ بx

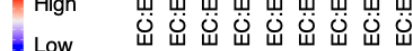
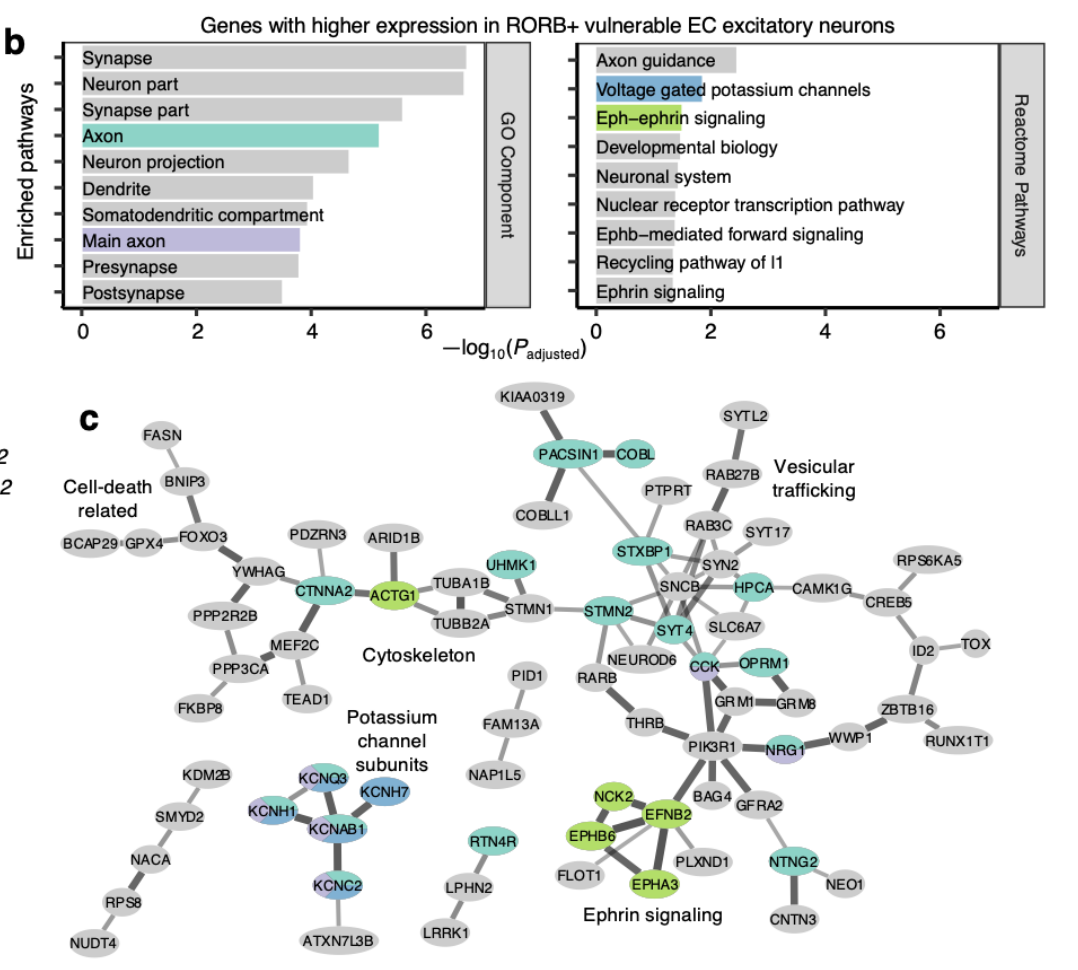

NUDT4

ATXN7LBB LRRK1
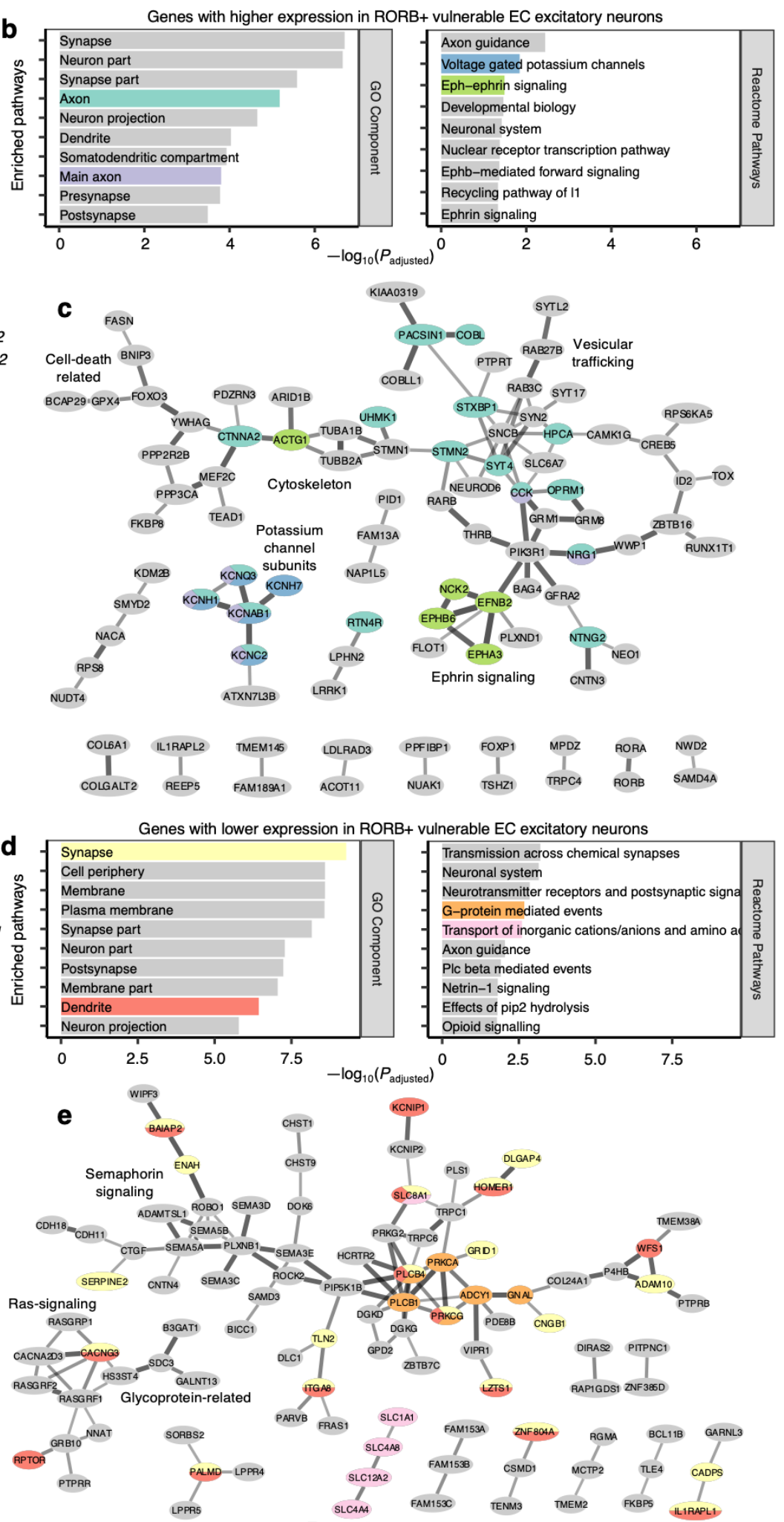

KCNG BRINP C1600152 STRSAS $\underset{\text { NRAA2 BRINPS VWA3A }}{\mid}$

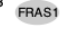
SLCAAB
SLCAA4
ransporters

LONRF1 1 KLLL3 
1233 Extended Data Fig. 2 | Expression of selected EC excitatory neuron subpopulation markers 1234 and pathway enrichment analysis of differentially expressed genes in selectively vulnerable 1235 EC excitatory neuron subpopulations. a, Expression heatmap of genes that are specifically 1236 expressed by four or fewer EC excitatory neuron subpopulations; "High" and "Low" relative 1237 expression reflect above- and below-average expression, respectively (see Methods). b-d, 1238 Enrichment analysis against Gene Ontology Cellular Component terms or Reactome Pathways 1239 (b,d) and functional association network analysis (c,e; see Methods) of genes with higher (b-c) 1240 or lower expression (d-e) in RORB+ vulnerable EC excitatory neurons, with selected terms 1241 highlighted by color. In panels $\mathbf{c}$ and $\mathbf{e}$, genes with stronger associations are connected by thicker 1242 lines, and genes without known associations are not shown. 
a

Differentially expressed genes

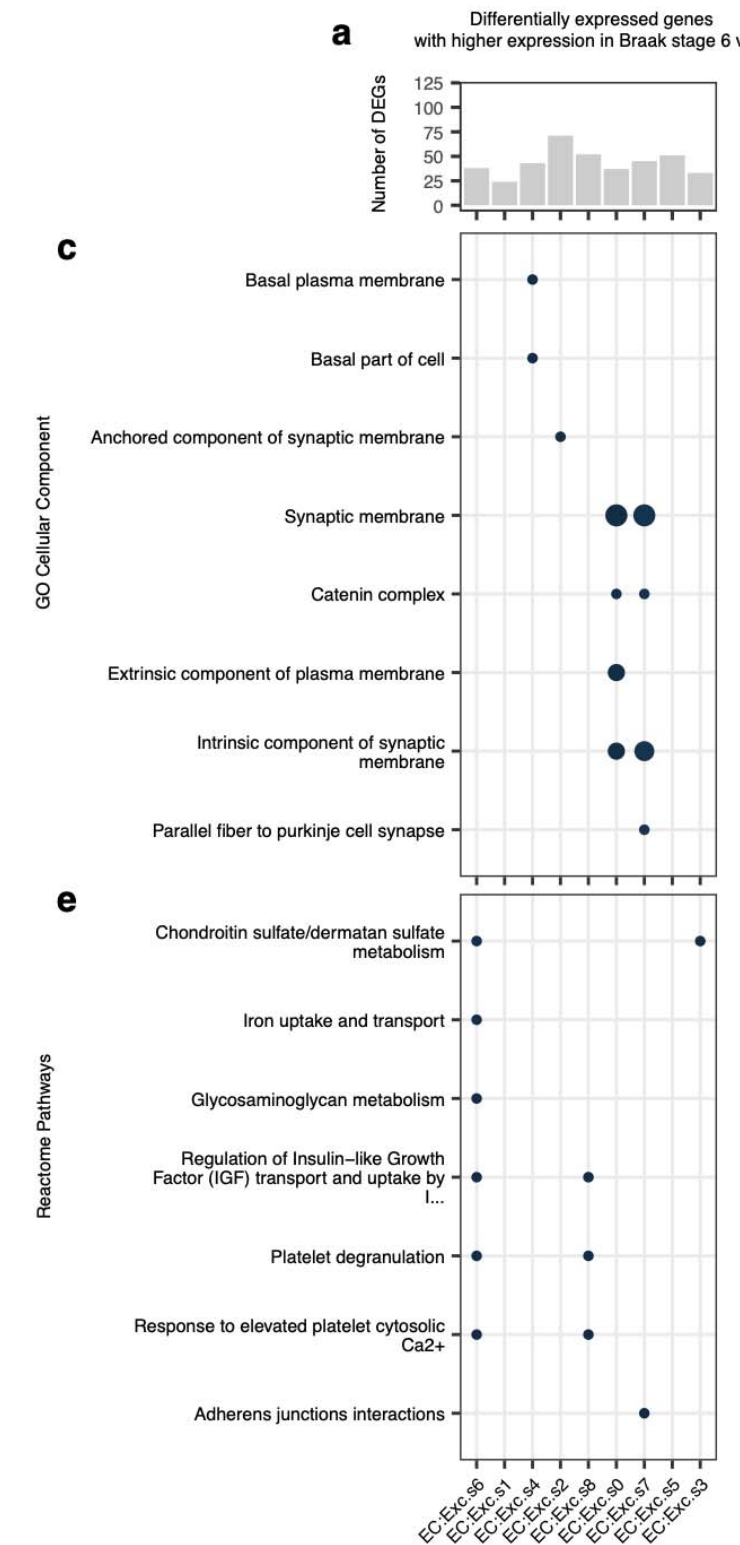

b Differentially expressed genes

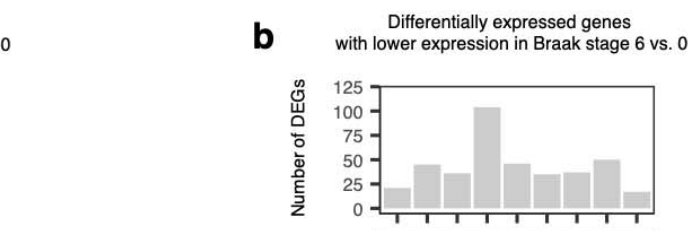

d

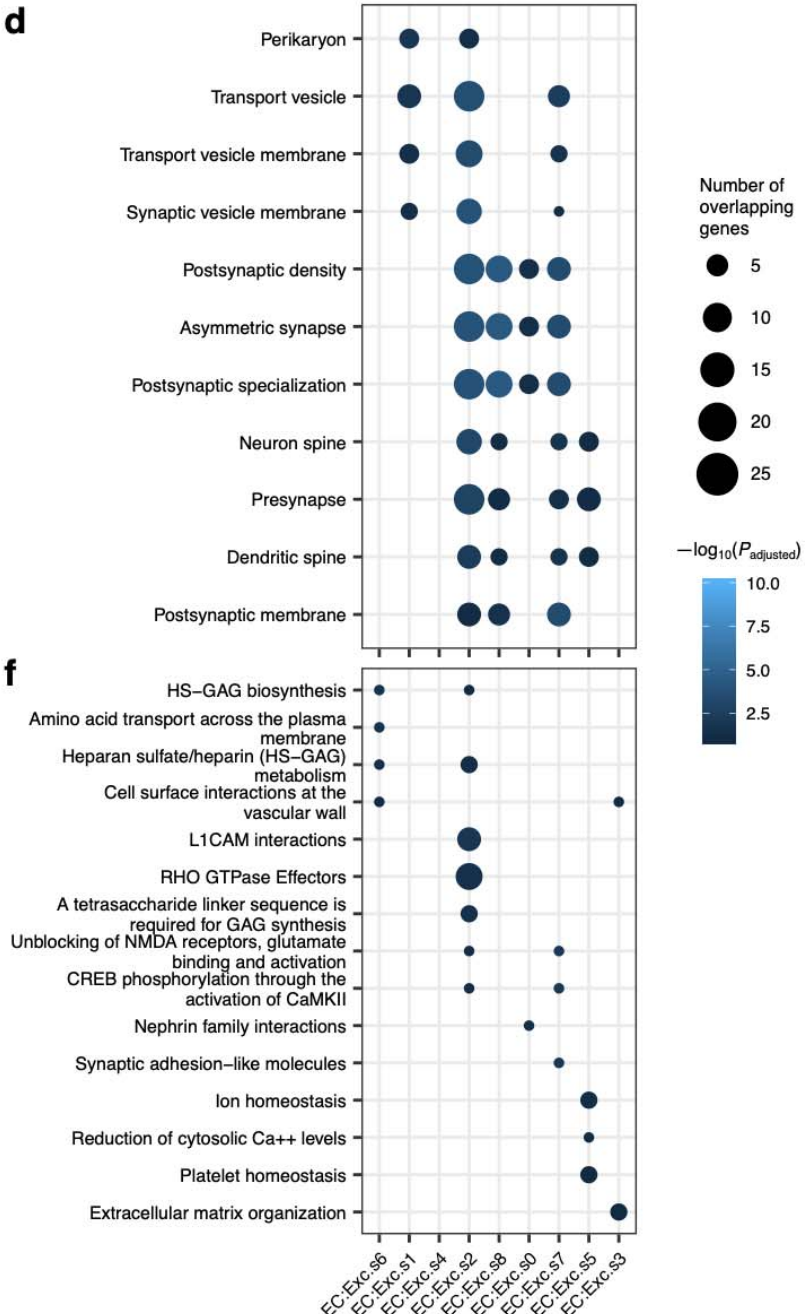

Extended Data Fig. 3 | Differential expression analysis across Braak stages for EC excitatory neuron subpopulations. a-b, Number of differentially expressed genes in EC excitatory neuron subpopulations with higher (a) or lower (b) expression in Braak stage 6 vs. Braak stage 0. c-f, Enrichment analysis against Gene Ontology Cellular Component terms (c-d) or Reactome Pathways (e-f) of differentially expressed genes in EC excitatory neuron subpopulations with higher $(\mathbf{c}, \mathbf{e})$ or lower $(\mathbf{d}, \mathbf{f})$ expression in Braak stage 6 vs. Braak stage 0 . 
EC and SFG excitatory neurons

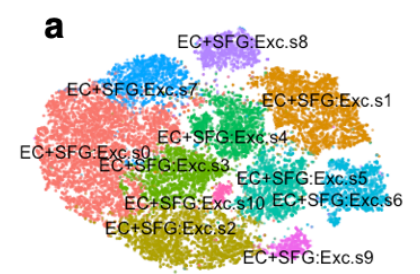

Case number
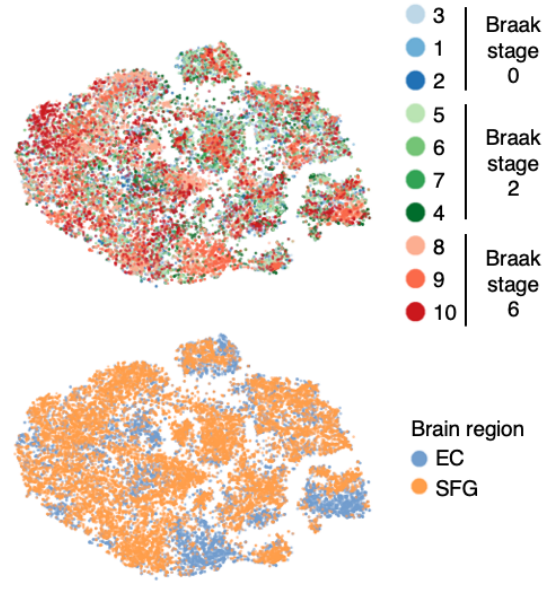

Brain region

EC

SFG

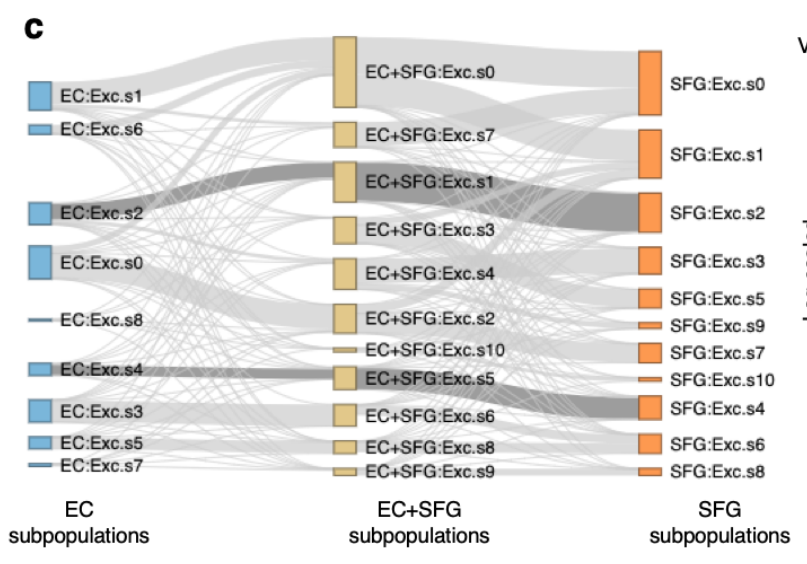

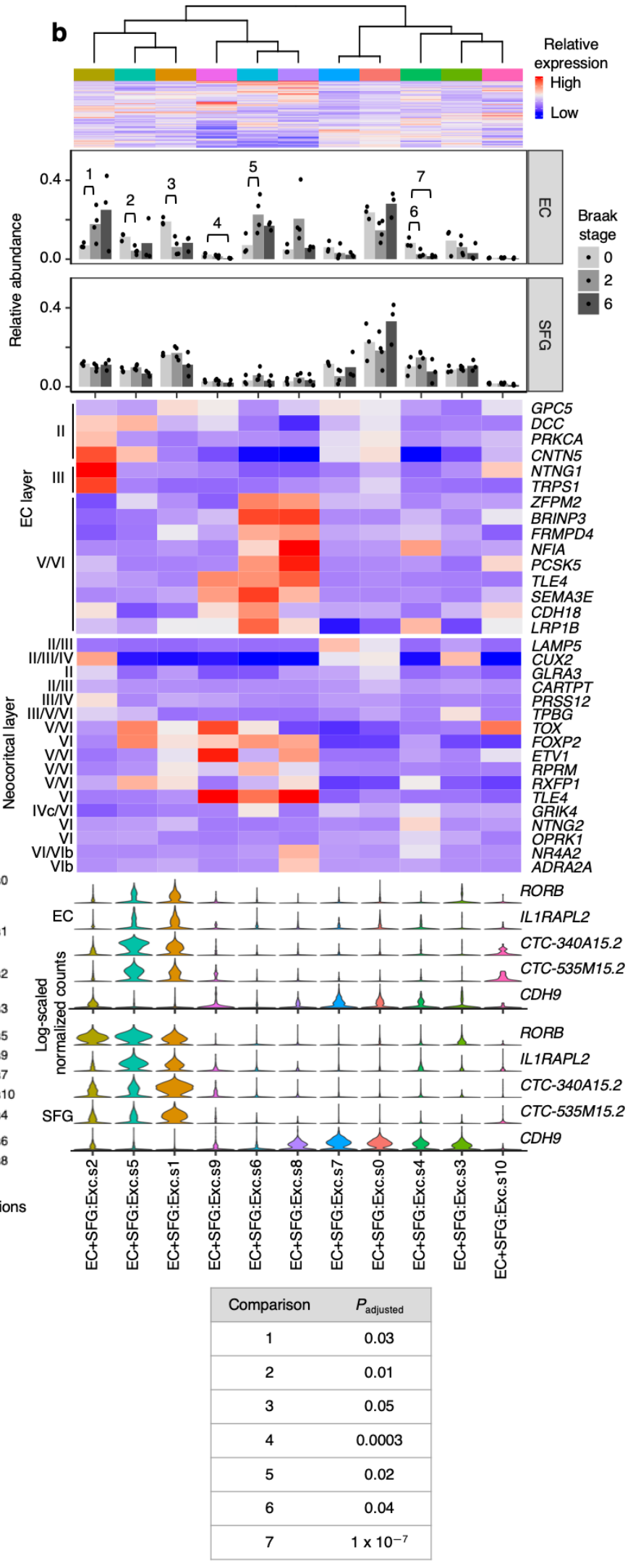


1253 Extended Data Fig. 4 | Alignment of EC and SFG maps homologous excitatory neuron 1254 subpopulations. a, tSNE projection of excitatory neurons from the EC and SFG in the joint 1255 alignment space, colored by subpopulation identity (top), individual of origin (middle), or brain 1256 region (bottom). b, Heatmap and hierarchical clustering of subpopulations and subpopulation marker expression (top subpanel); "High" and "Low" relative expression reflect above- and below-average expression, respectively (see Methods). Relative abundance of subpopulations across Braak stages (second and third subpanels). Expression heatmap of EC layer-specific genes identified from Ramsden et al. ${ }^{34}$ (fourth subpanel). Expression heatmap of neocortical layerspecific genes from Lake et al. ${ }^{19}$ (fifth subpanel). Expression of selectively vulnerable EC excitatory neuron subpopulation markers by excitatory neurons in the EC (sixth subpanel) or SFG (bottom subpanel). Significant beta regression $P$ values (adjusted for multiple testing) are shown in a table at the bottom of the panel. c, Sankey diagram connecting subpopulation identity of excitatory neurons in the EC alignment space and the SFG alignment space to subpopulation identity in the EC+SFG alignment space. The links connecting EC:Exc.s2 and EC:Exc.s4 to 1268 SFG:Exc.s2 and SFG:Exc.s4, respectively, are highlighted. 


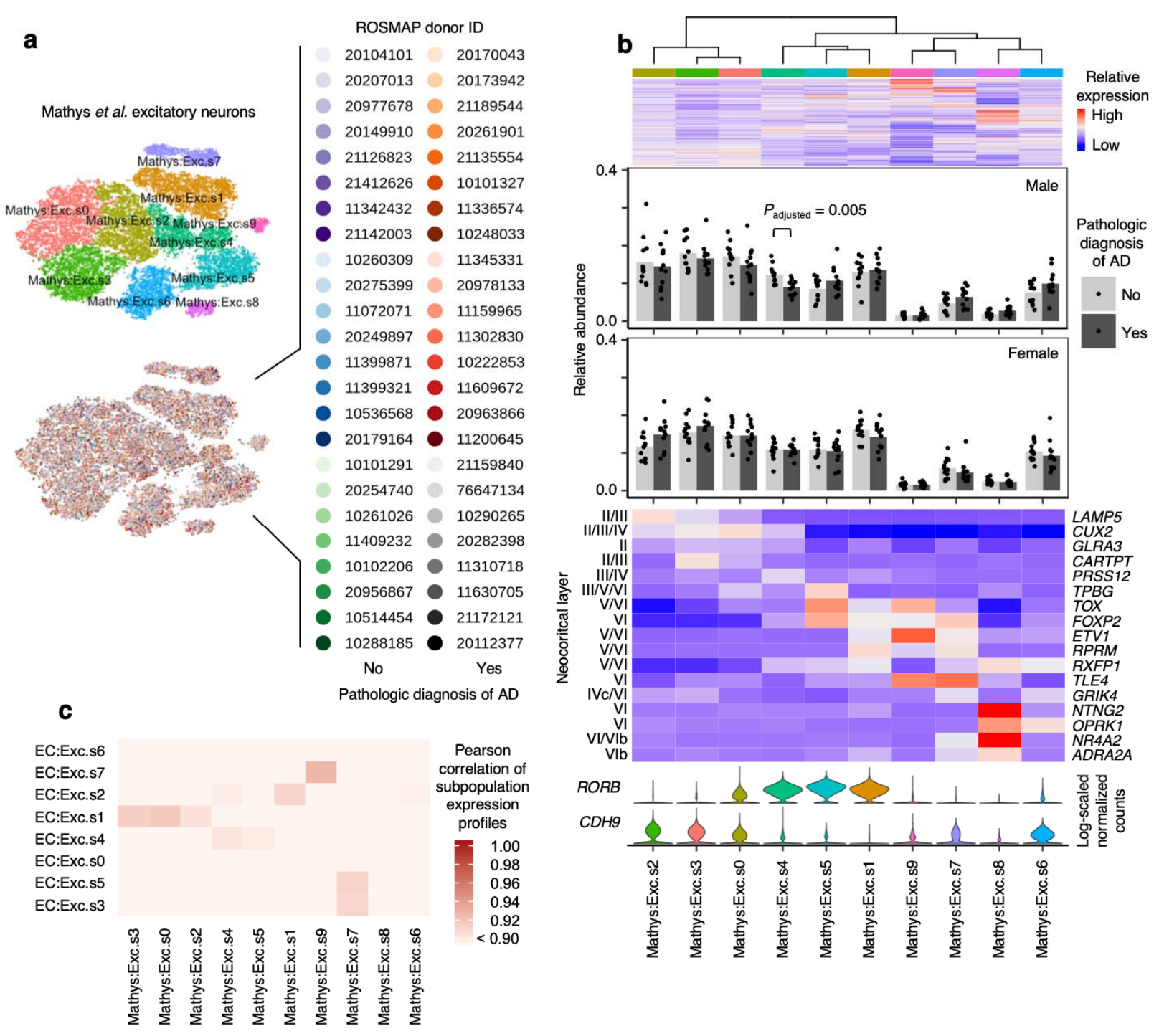

\section{Extended Data Fig. 5 | Cross-sample alignment of excitatory neurons from Mathys et al.} recapitulates selective vulnerability in a RORB-expressing subpopulation. a, tSNE projection of excitatory neurons from Mathys et l. $^{21}$ in the alignment space, colored by subpopulation identity (top) or individual of origin (bottom). b. Heatmap and hierarchical clustering of subpopulations and subpopulation marker expression (top subpanel); "High" and "Low" relative expression reflect above- and below-average expression, respectively (see Methods). Relative abundance of subpopulations in in AD cases vs. controls, separated by sex (second and third subpanels). Expression heatmap of neocortical layer-specific genes from Lake et al. ${ }^{19}$ (fourth subpanel). Expression of selectively vulnerable EC excitatory neuron subpopulation markers (bottom subpanel). c, Heatmap of Pearson correlation between the gene expression profiles of excitatory neuron subpopulations from the EC vs. those from the prefrontal cortex in Mathys et al. ${ }^{21}$ 
a

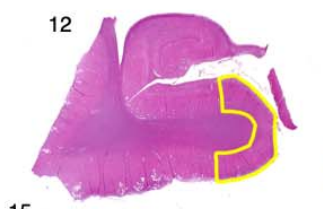

15

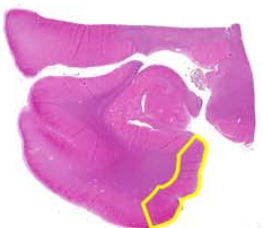

5
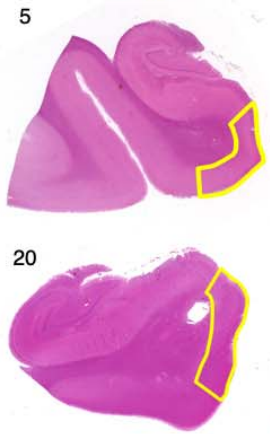

25 .

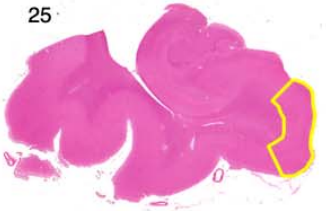

27

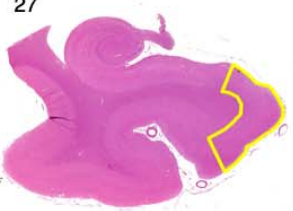

30

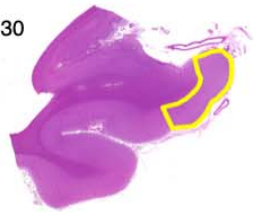

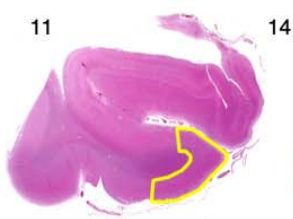

16

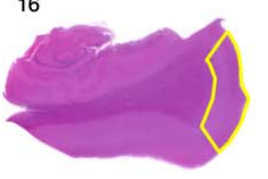

60
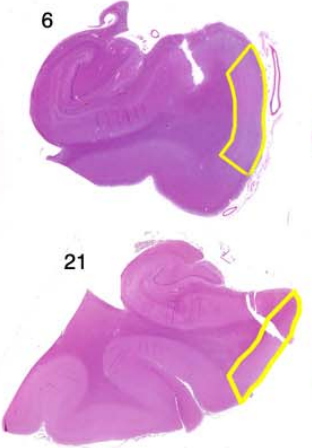

24

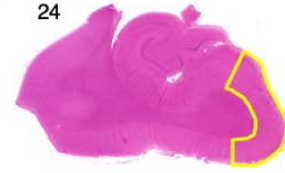

28
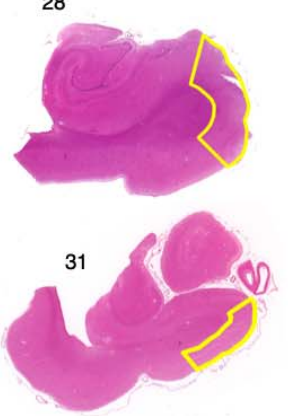

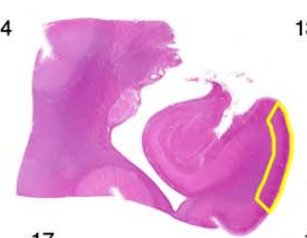

17

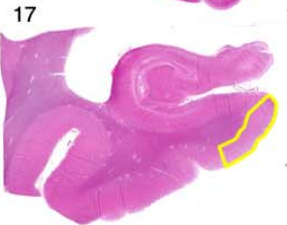

7

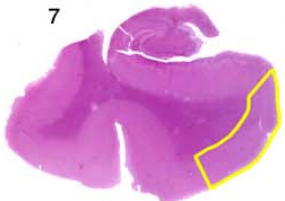

22
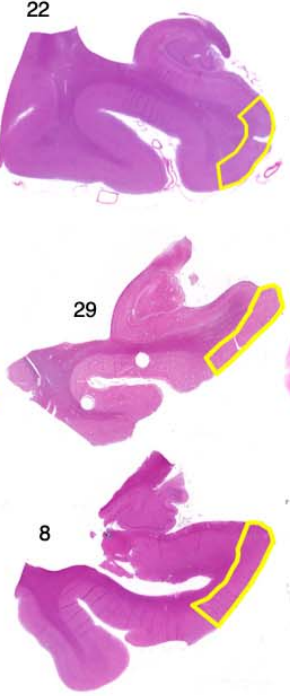

3

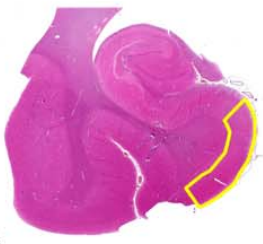

18
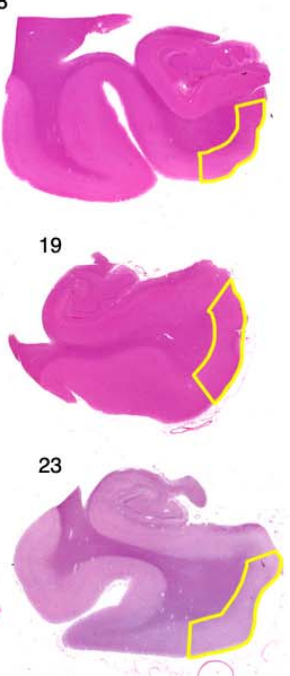

26
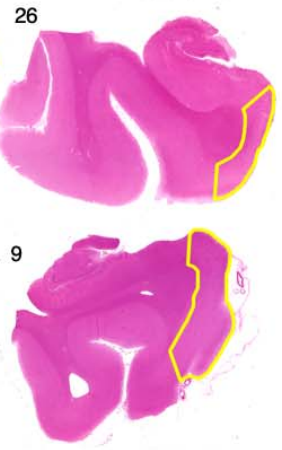
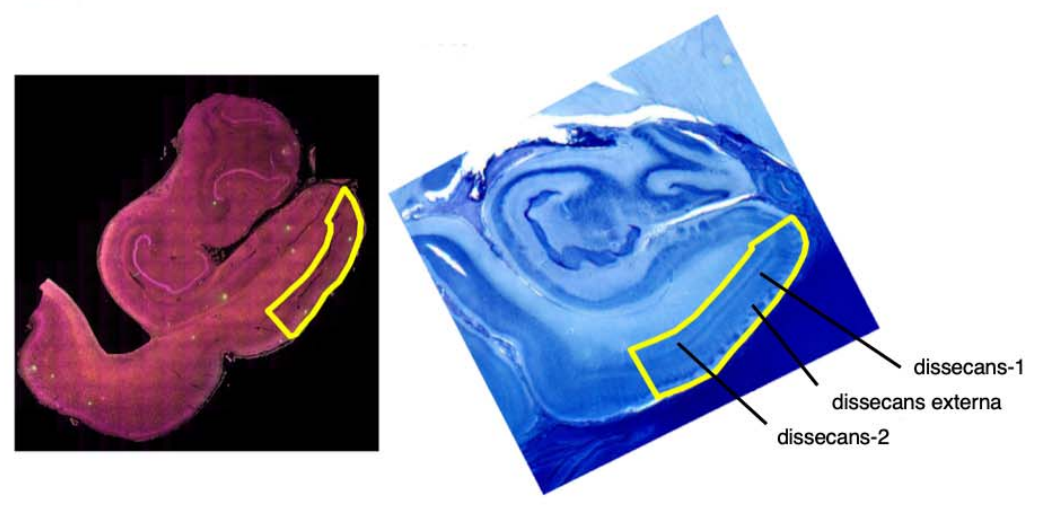
1284 Extended Data Fig. 6 | Delineation of the EC for each case used in immunofluorescence 1285 validation. a, The borders of the caudal EC delineated on sections stained with hematoxylin and 1286 eosin (H\&E) for all 26 cases used in immunofluorescence validation (Table 1). b, Borders of the EC were determined with the aid of 400 um thick serial coronal sections of whole-brain 1288 hemispheres stained with gallocyanin (see Methods). Each H\&E section (left) along with its 1289 corresponding immunofluorescence image (middle) was aligned to the most approximate gallocyanin section (right), in which the the dissecans layers (diss-1, diss-2, and diss-ext) characteristic of the caudal EC were easier to visualize. This was then used to guide delineation of the EC on the H\&E and immunofluorescence sections. For more details on the cytoarchitectonic definitions used to define the caudal EC, please consult Heinsen et al. ${ }^{42}$. 

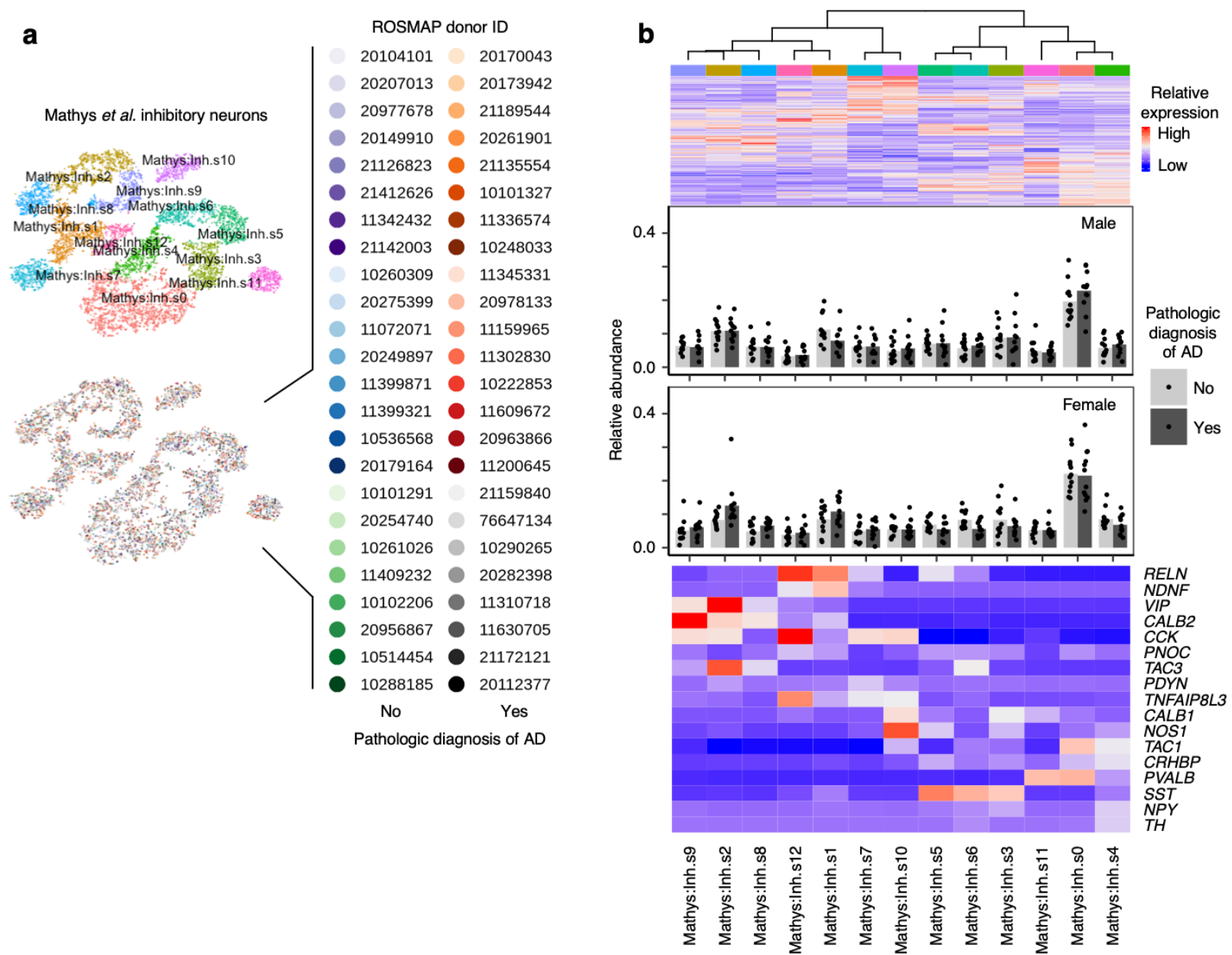

Extended Data Fig. 7 | Inhibitory neurons from Mathys et al. also do not show differences in resilience or vulnerability to AD. a, tSNE projection of inhibitory neurons from Mathys et $a l^{21}$ in the alignment space, colored by subpopulation identity (top) or individual of origin (bottom). b, Heatmap and hierarchical clustering of subpopulations and subpopulation markers (top subpanel); "High" and "Low" relative expression reflect above- and below-average expression, respectively (see Methods). Relative abundance of subpopulations in in AD cases vs. controls, separated by sex (second and third subpanels). Expression heatmap of inhibitory neuron subtype markers from Lake et al. ${ }^{19}$ (bottom subpanel). 


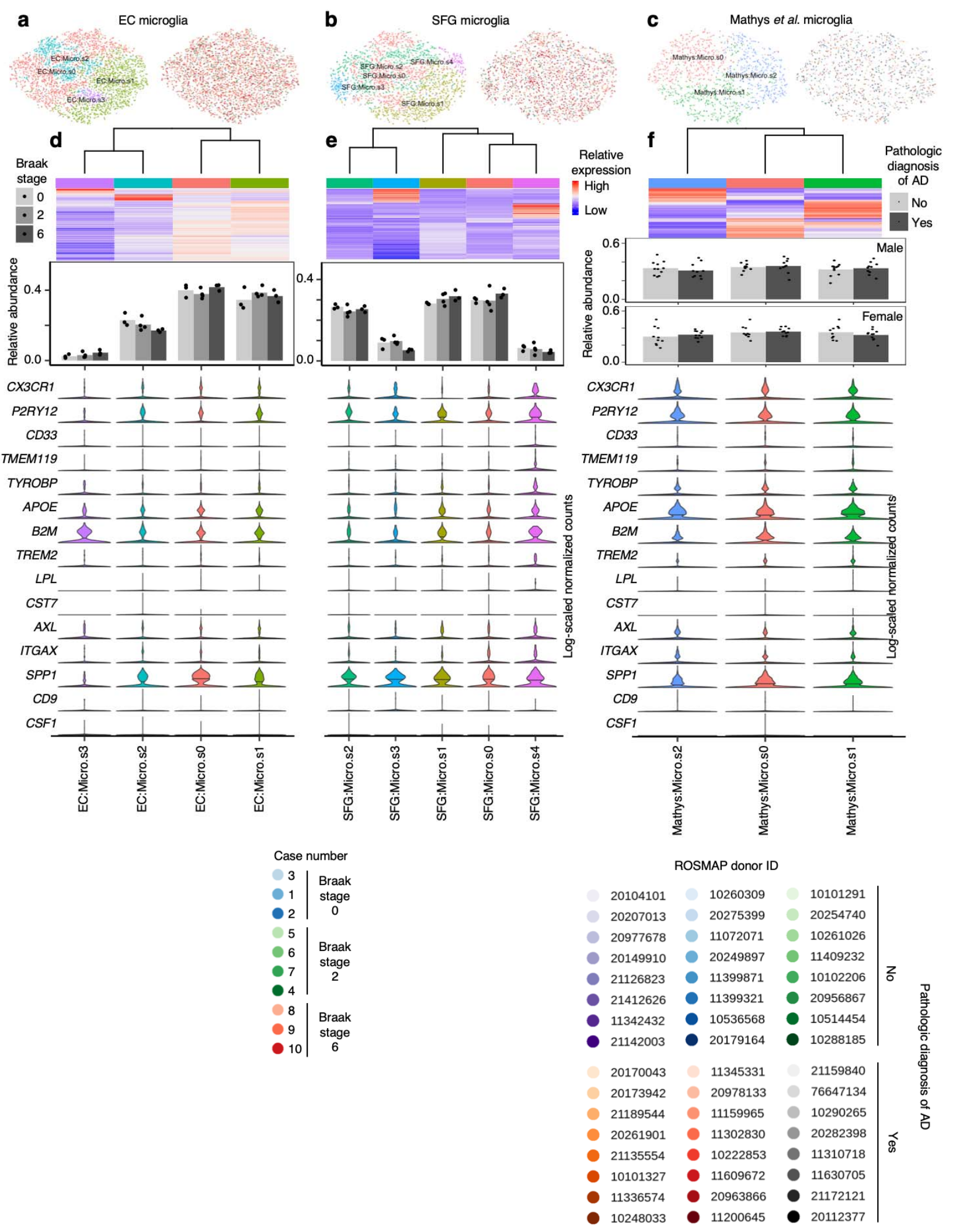


1306 Extended Data Fig. 8 | Subclustering of microglia does not sufficiently resolve disease 1307 associated microglia signature. a-c, tSNE projection of astrocytes from the EC (a), SFG (b), 1308 and Mathys et $a .^{21}$ (c) in their respective alignment spaces, colored by subpopulation identity 1309 (left) or individual of origin (right). d-f, Heatmap and hierarchical clustering of subpopulations 1310 and subpopulation marker expression (top subpanels); "High" and "Low" relative expression 1311 reflect above- and below-average expression, respectively (see Methods). Relative abundance of 1312 subpopulations across Braak stages in the EC and SFG or between AD cases vs. controls in 1313 Mathys et al. ${ }^{21}$ (middle subpanels). Expression of disease associated microglia markers, with 1314 median expression level marked by line (bottom subpanels). 


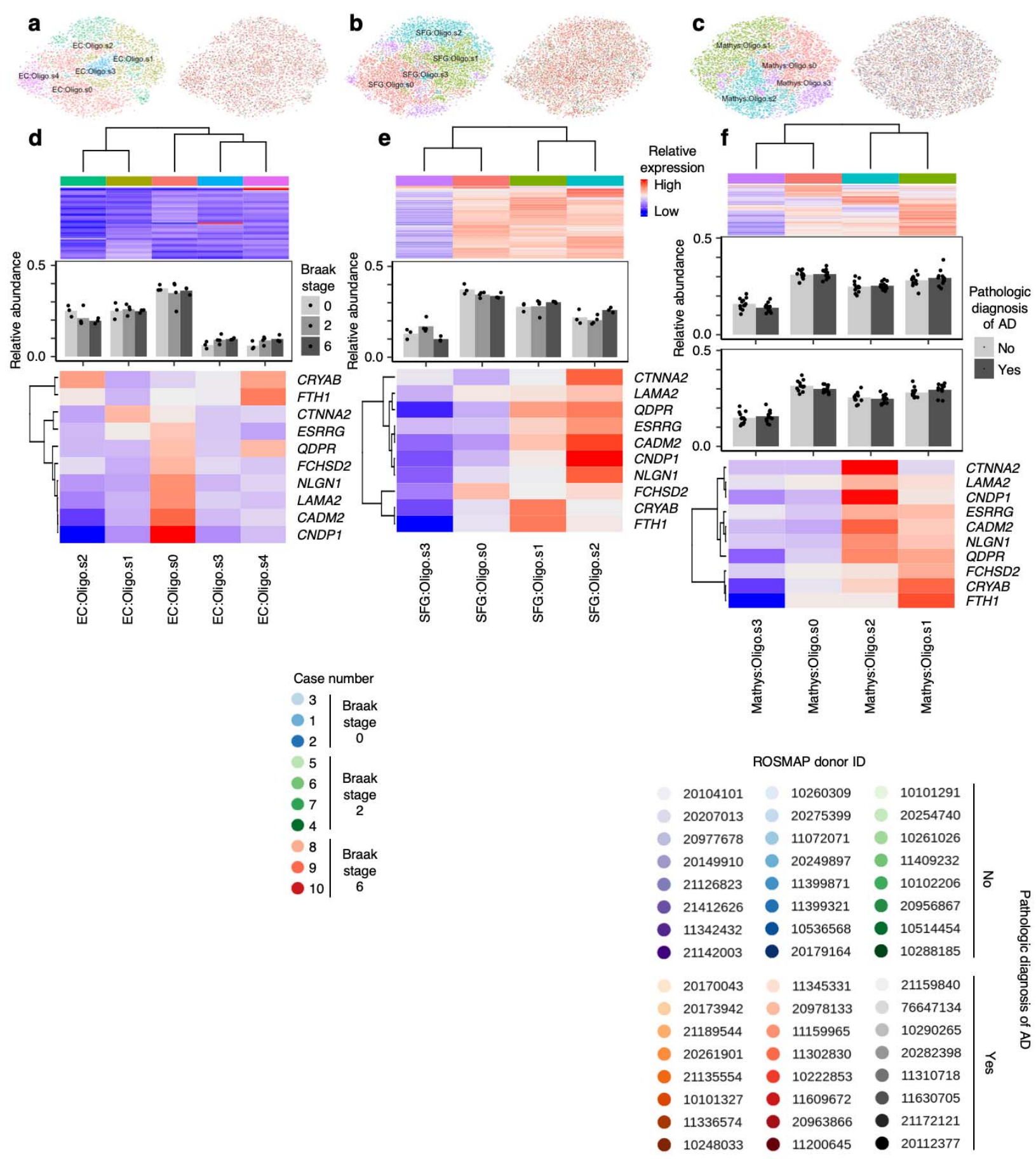

Extended Data Fig. 9 | Subclustering of oligodendrocytes identifies subpopulations with higher expression of AD-associated oligodendrocyte markers from Mathys et al. a-c, tSNE projection of oligodendrocytes from the EC (a), SFG (b), and Mathys et al. ${ }^{21}$ (c) in their respective alignment spaces, colored by subpopulation identity (left) or individual of origin (right). d-f, Heatmap and hierarchical clustering of subpopulations and subpopulation marker expression (top subpanels); "High" and "Low" relative expression reflect above- and belowaverage expression, respectively (see Methods). Relative abundance of subpopulations across Braak stages in the EC and SFG or between AD cases vs. controls in Mathys et al. ${ }^{21}$ (middle 
bioRxiv preprint doi: https://doi.org/10.1101/2020.04.04.025825; this version posted August 23, 2020. The copyright holder for this preprint (which was not certified by peer review) is the author/funder. All rights reserved. No reuse allowed without permission.

1325 subpanels). Relative expression of AD-associated oligodendrocyte subpopulation markers from 1326 Mathys et al. ${ }^{21}$ (bottom subpanels). 

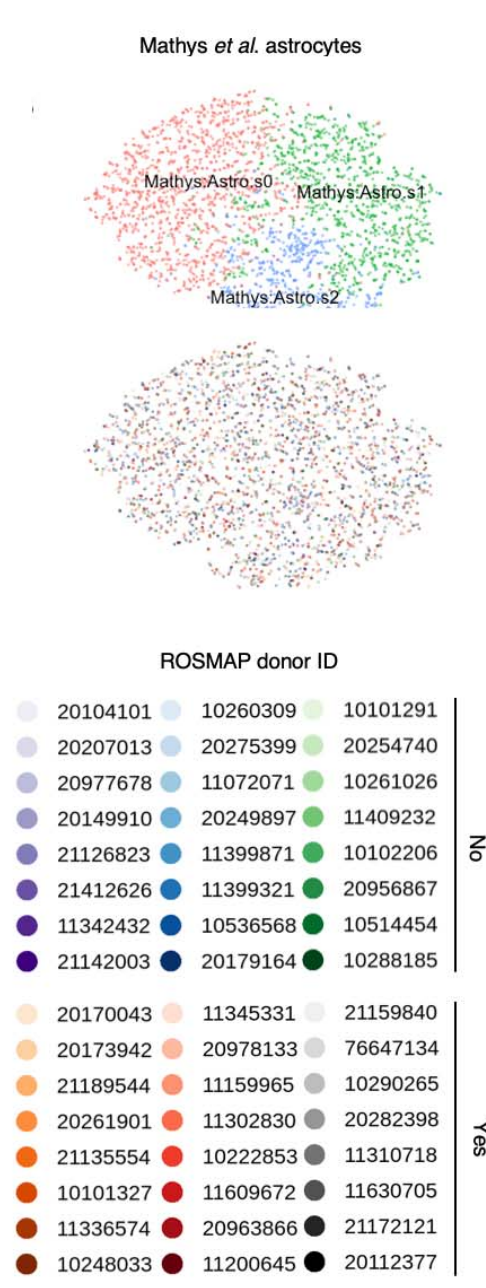

ROSMAP donor ID

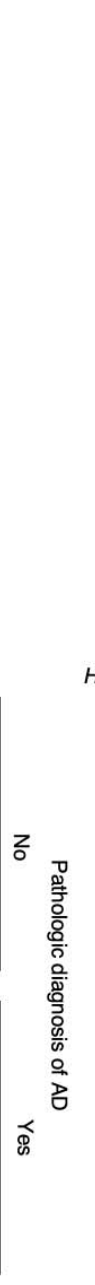

b
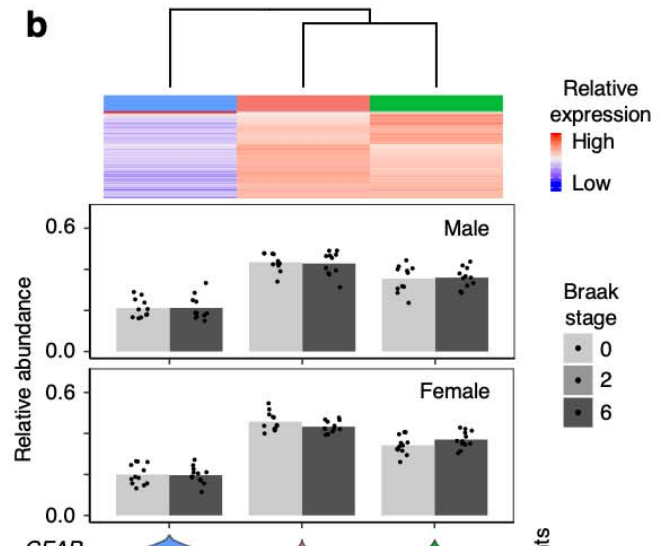

Braak

stage

- 0

- 6 et al. are highly similar to those from the EC and SFG. a, tSNE projection of astrocytes from Mathys $e t$ al. $^{21}$ in the alignment subspace, colored by subpopulation identity (top) or individual of origin (bottom). b. Heatmap and hierarchical clustering of subpopulations and subpopulation marker expression (top subpanel); "High" and "Low" relative expression reflect above- and below-average expression, respectively (see Methods). Relative abundance of subpopulations in in $\mathrm{AD}$ cases vs. controls, separated by sex (middle subpanels). Expression of genes associated with reactive astrocytes, with median expression level marked by line (bottom subpanel). c, Enrichment analysis of overlap between differentially expressed genes in astrocytes with high GFAP expression from Mathys et al. ${ }^{21}$ vs. differentially expressed genes in astrocytes with high GFAP expression from the EC and SFG; the number of genes in each gene set and the number of 
bioRxiv preprint doi: https://doi.org/10.1101/2020.04.04.025825; this version posted August 23, 2020. The copyright holder for this preprint (which was not certified by peer review) is the author/funder. All rights reserved. No reuse allowed without permission.

1340 overlapping genes are shown in parentheses, and the hypergeometric test p-values are shown 1341 without parentheses.

1342 


\title{
1343 SUPPLEMENTARY TABLE LEGEND
}

\section{4}

\begin{abstract}
Supplemental Table 1 | Genes differentially expressed by selectively vulnerable excitatory neurons compared to all other excitatory neurons in the EC. The column "gene" contains official gene symbols of differentially expressed genes, "logFC" contains the associated $\log _{2}$ fold-change, " $\log$ CPM" contains the $\log _{2}$-transformed normalized counts of transcripts mapping to the gene averaged across all conditions, "F" contains the value of the quasi-likelihood Fstatistic (see edgeR documentation) used to determine differential expression, "Pvalue" contains the raw $P$ values associated with the quasi-likelihood F-test, "FDR" contains $P$ values adjusted for multiple testing using the Benjamini-Hochberg method.
\end{abstract}

